LU TP 00-42

hep-ph/0010012

October 2000

\title{
QCD Radiation off Heavy Particles
}

\author{
E. Norrbin' and T. Sjöstrand \{ \\ Department of Theoretical Physics, \\ Lund University, \\ Sölvegatan $14 A$, \\ S-223 62 Lund, Sweden
}

\begin{abstract}
We study QCD radiation in decay processes involving heavy particles. As input, the first-order gluon emission rate is calculated in a number of reactions, and comparisons of the energy flow patterns show a non-negligible process dependence. To proceed further, the QCD parton shower language offers a convenient approach to include multi-gluon emission effects, and to describe exclusive event properties. An existing shower algorithm is extended to take into account the process-dependent mass, spin and parity effects, as given by the matrix element calculations. This allows an improved description of multiple gluon emission effects off $b$ and $t$ quarks, and also off nonstandard particles like squarks and gluinos. Phenomenological applications are presented for bottom production at LEP, Higgs particle decay to heavy flavours, top production and decay at linear colliders, and some simple supersymmetric processes.
\end{abstract}

${ }^{1}$ emanuel@thep.lu.se

${ }^{2}$ torbjorn@thep.lu.se 


\section{Introduction}

Heavy particles often tend to be in the focus of particle physics research. The search for new physics at the high-energy frontier is an obvious example. Not only are the hypothetical new heavy states here of interest, but often their detection relies on decay chains that involve heavy flavours. Here the b quark is the prime example. By secondary vertices or semileptonic decays it can be tagged. It is a main decay product of top, where the $\mathrm{b}$ tag is a standard requirement for top identification above background at hadron colliders. A light Standard Model Higgs $\left(m_{\mathrm{h}} \lesssim 140 \mathrm{GeV}\right)$ predominantly decays to b $\bar{b}$, and for any Higgs state the $\mathrm{b} \overline{\mathrm{b}}$ branching ratio is a key parameter in pinning down its nature. These tagging and identification aspects also appear in other scenarios beyond the standard model.

Many of the potentially new particles carry colour, like squarks, gluinos and leptoquarks. Also colourless states often decay to quarks. Therefore QCD radiation is unavoidable, and the large value of the $\alpha_{\mathrm{s}}$ coupling implies that such radiation can be quite profuse. It is well-known, e.g. from LEP, that fixed-order perturbation theory fails to describe QCD radiation effects off light quarks: the rate of a few well separated jets can be reproduced, but the internal structure of these jets requires the resummation of multiple-gluon effects. The most successful method for achieving such a resummation is to apply the parton-shower language, where explicit final states can be generated, with full respect for energy-momentum conservation and other constraints. By introducing some low fixed cut-off scale $Q_{0} \approx 1 \mathrm{GeV}$, the very soft and nonperturbative régime of QCD can be factored off and put in a universal hadronization description, such as the Lund string fragmentation model [1]. With this approach, it is possible to obtain a quite accurate description of essentially all hadronic final-state properties of $\mathrm{e}^{+} \mathrm{e}^{-}$annihilation events. The physics is more complicated in a hadron collider environment, with additional effects e.g. from initial-state QCD radiation and underlying events, so the level of ambition may have to be set accordingly. However, this applies to all conceivable descriptions, so again we expect a realistic description of jets to be most easily achieved with the help of the parton-shower language.

Heavy quarks radiate less than light ones in the soft-gluon region, so in some respects QCD multiple-emission corrections are not as crucial. The b is sufficiently light that it still radiates profusely, but top and supersymmetric particles may be heavy enough that multiple-gluon emission effects are limited. Furthermore, for particles that are very short-lived, the width will provide a cut-off on soft-gluon emission [2]. So for new-particle searches, higher-order QCD effects may be less of an issue. However, whenever higher precision is required, like for a mass determination of a new state, it may still be necessary to model whatever soft-gluon emission effects there are. And, of course, the decays of these new states will bring further radiation, e.g. like $t \rightarrow \mathrm{bW}^{+}$, where the radiation off the $\mathrm{t}$ and off the $\mathrm{b}$ become intertwined, and where width effects may become important for short-lived particles. The aim of the current article is precisely to improve the description of gluon radiation off b and c quarks as well as off heavier objects, in order to allow higher-precision physics studies.

The starting point is the calculation of a large number of first-order matrix elements, for gluon emission in decay processes, often generalizing on results already found in the literature. The processes thereby covered include gluon emission e.g. in $\gamma^{*} / \mathrm{Z}^{0} / \mathrm{h}^{0} / \mathrm{A}^{0} \rightarrow \mathrm{b} \overline{\mathrm{b}}$ (with differences between a vector, axial vector, scalar and pseudoscalar source of the b's), $\mathrm{t} \rightarrow \mathrm{bW}^{+}$, and various supersymmetric reactions. Quite apart from the implemen- 
tation in the shower framework, these calculations can also be used to assess the degree of (non)universality, i.e. the dependence on the colour and spin structure of the processes under identical kinematical conditions. In particular, we will show that the 'dead cone' picture can be quite misleading for the emission of more energetic gluons.

These matrix elements are taken as input to an improved version of an existing shower algorithm [3], which primarily has been used for radiation off lighter quarks and gluons. This shower algorithm tends to slightly overestimate the amount of gluon radiation for $\mathrm{e}^{+} \mathrm{e}^{-} \rightarrow \gamma^{*} / \mathrm{Z}^{0} \rightarrow \mathrm{q} \overline{\mathrm{q} g}$ when $\mathrm{q}$ is massless, so a simple rejection procedure can be used to match the hardest emission to the massless matrix elements. The radiation off heavier quarks is underestimated in the collinear region, however. Or, put differently, the dead cone [4] effects are exaggerated. In order to allow a corresponding approach for heavy flavours, the evolution variable therefore has been modified to bring up the emission rate. It therefore becomes possible to use the process-specific matrix elements as rejection factors also here, and furthermore to do it in all stages of the shower.

The one place where precision tests are possible is in studies of b production at LEP1 [5]. We therefore study this topic in detail here, and use it as a test bed for different possible variations of the basic shower algorithm. Other studies include Higgs, top and supersymmetric particle production and decay. Furthermore, we also include some plots to illustrate differences between gluon radiation off sources of different spin.

While not the main thrust of this article, we note that new data on gluon branching to heavy flavours, $\mathrm{g} \rightarrow \mathrm{c} \overline{\mathrm{c}}$ and $\mathrm{g} \rightarrow \mathrm{b} \overline{\mathrm{b}}$, has been presented by the LEP collaborations [5]. We therefore also discuss what implications this might have for the shower algorithm, and whether the data could be accommodated by reasonable modifications.

The plan of the article is as follows. In Section 2, the existing older shower algorithm is explained, together with a few intermediate variants thereof. The new approach is described in Section 3. A survey of the matrix-element calculations and the consequent radiation patterns is given in Section 4. Some applications are then presented in Section 5. The separate topic of gluon splitting is discussed in Section 6. Finally, a summary and outlook is given in Section 7.

\section{Previous Models}

Several shower algorithms have been proposed in the literature. Today the three most commonly used ones probably are those found in Pythia/Jetset [3, 6], HeRwig [7, 8] and Ariadne [9]. The studies in this article will be based on the former one.

The PYThia final-state shower consists of an evolution in the squared mass $m^{2}$ of a parton. That is, emissions are ordered in decreasing mass of the radiating parton, and the Sudakov form factor [10] is defined as the no-emission rate in the relevant mass range. Such a choice is not as sophisticated as the angular one in HERWIG or the transverse momentum one in ARIADNE, but usually the three tend to give similar results for $\mathrm{e}^{+} \mathrm{e}^{-}$ annihilation events. An exception, where small but significant differences were found, is the emission of photons in the shower [11]. In general, comparisons between the three are helpful in estimating a range of theoretical uncertainty, in interpretations of existing data or in predictions for the future.

One of the advantages of the PYTHIA algorithm is that a mapping between the parton-shower and matrix-element variables is rather straightforward to $\mathcal{O}\left(\alpha_{\mathrm{s}}\right)$ for massless quarks, and that already the basic shower populates the full phase space region very closely the same way as the matrix element. It is therefore possible to introduce a simple 
correction to the shower to bring the two into agreement. Also in ArIADNE the massless matrix-element matching is straightforward. By contrast, the HERWIG angular-ordered approach does not automatically cover the full $q \bar{q} g$ phase space, which means that a subset of three-jet events are not generated at all. This leads to problems in the description of LEP data, that were overcome by separately adding the missing class of three-jet events [12], in addition to matching to the matrix elements in the allowed region. More recently, a similar approach has been applied to top decay [13], where again HeRwIG did not populate the full phase space.

\subsection{The massless shower}

In addition to mass, the other main variable in the PутніA shower is $z$, as used in the splitting kernels. It is defined as the energy fraction taken by the first daughter in the CM frame of the event. That is, in a branching $a \rightarrow b+c, E_{b}=z E_{a}$ and $E_{c}=(1-z) E_{a}$. In the original choice of $z$, which is done at the same time as $m_{a}$ is selected, the $b$ and $c$ masses are not yet known, and are therefore imagined massless, also in cases where either of them is known to have a non-vanishing on-shell mass. A cut-off scale $m_{\min }=Q_{0} \approx 1 \mathrm{GeV}$ is used to constrain the allowed phase space, so that only branchings with $m_{a}>m_{\text {min }}$ are allowed. For a massive quark, the cut-off is shifted to

$$
m_{a, \min }=\sqrt{m_{a}^{2}+\frac{Q_{0}^{2}}{4}}+\frac{Q_{0}}{2}
$$

The allowed $z$ range, $z_{-}<z<z_{+}$, then becomes

$$
z_{ \pm}=\frac{1}{2}\left\{1 \pm \beta_{a} \Theta\left(m_{a}-m_{a, \min }\right)\right\}
$$

with $\beta_{a}=\left|\mathbf{p}_{a}\right| / E_{a}$ the $a$ velocity and $\Theta(x)$ the step function.

At a later stage of the evolution, when $m_{b}$ and $m_{c}$ are being selected, possibly well above $Q_{0}$, the previously found $z$ may be incompatible with these masses. The adopted solution is to take into account mass effects by reducing the magnitude of the threemomenta $\mathbf{p}_{b}=-\mathbf{p}_{c}$ in the rest frame of $a$. Expressed in terms of four-momenta in an arbitrary frame, this is equivalent to

$$
\begin{aligned}
& p_{b}=\left(1-k_{b}\right) p_{b}^{(0)}+k_{c} p_{c}^{(0)}, \\
& p_{c}=\left(1-k_{c}\right) p_{c}^{(0)}+k_{b} p_{b}^{(0)},
\end{aligned}
$$

where $p_{b}^{(0)}$ and $p_{c}^{(0)}$ are the original massless momenta and $p_{b}$ and $p_{c}$ the modified massive ones. The parameters $k_{b}$ and $k_{c}$ are found from the constraints $p_{b}^{2}=m_{b}^{2}$ and $p_{c}^{2}=m_{c}^{2}$ :

$$
k_{b, c}=\frac{m_{a}^{2}-\lambda_{a b c} \pm\left(m_{c}^{2}-m_{b}^{2}\right)}{2 m_{a}^{2}}
$$

with

$$
\lambda_{a b c}=\sqrt{\left(m_{a}^{2}-m_{b}^{2}-m_{c}^{2}\right)^{2}-4 m_{b}^{2} m_{c}^{2}} .
$$

The relation between the preliminary and final energy sharing thus is given by

$$
z^{\prime}=\frac{E_{b}}{E_{a}}=\left(1-k_{b}\right) z+k_{c}(1-z)=\frac{m_{a}^{2}-\lambda_{a b c}+m_{b}^{2}-m_{c}^{2}}{2 m_{a}^{2}}+\frac{\lambda_{a b c}}{m_{a}^{2}} z,
$$



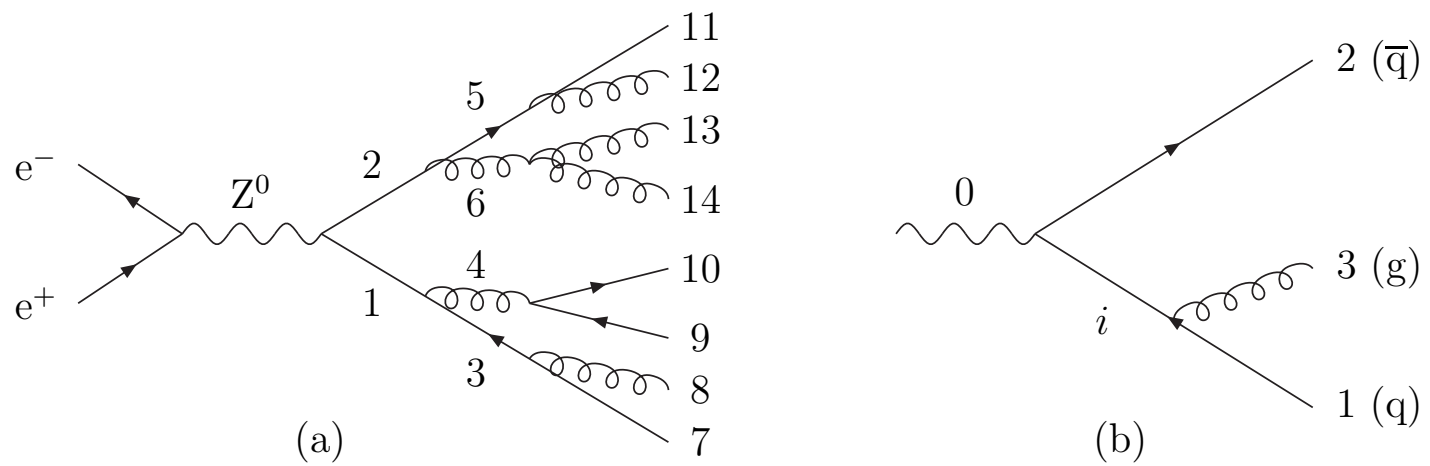

Figure 1: Example of showers, with the notations used in the text. (a) A generic shower. (b) A shower giving a three-jet event.

with $z=E_{b}^{(0)} / E_{a}$ as above. The transverse momentum $p_{\perp}$ of $b$ and $c$ with respect to the $a$ direction is given by

$$
p_{\perp}^{2}=\frac{\lambda_{a b c}^{2}}{m_{a}^{2}}\left\{\frac{z(1-z)}{\beta_{a}^{2}}-\frac{1-\beta_{a}^{2}}{4 \beta_{a}^{2}}\right\},
$$

but is approximated by $p_{\perp}^{2} \approx z(1-z) m_{a}^{2}$ when used as argument in $\alpha_{\mathrm{s}}\left(p_{\perp}^{2}\right)$ for the shower.

Angular ordering is not automatic, but is implemented by vetoing emissions that don't correspond to decreasing opening angles. The opening angle of a branching $a \rightarrow b+c$ is calculated approximately as

$$
\theta \approx \frac{p_{\perp b}}{E_{b}}+\frac{p_{\perp c}}{E_{c}} \approx \sqrt{z(1-z)} m_{a}\left(\frac{1}{z E_{a}}+\frac{1}{(1-z) E_{a}}\right)=\frac{1}{\sqrt{z(1-z)}} \frac{m_{a}}{E_{a}}
$$

The procedure thus is the following, Fig. 1a. In the $\gamma^{*} / \mathrm{Z}^{0}$ decay, the two original partons 1 and 2 are produced, back-to-back in the rest frame of the pair. In a first step, they are evolved downwards from a maximal mass equal to the CM energy, with the restriction that the two masses together should be below this CM energy. When the two branchings are found, they define $m_{1}$ and $m_{2}$ and the $z$ values of $1 \rightarrow 3+4$ and $2 \rightarrow 5+6$. These branchings obviously have smaller opening angles than the $180^{\circ}$ one between 1 and 2, so no angular-ordering constraints appear here. A matching procedure to the matrix element is used to correct the branchings, however, as will be described below. In subsequent steps, a pair of partons like 3 and 4 are evolved in parallel, from maximum masses given by the smaller of the mother (1) mass and the respective daughter (3 or 4 ) energy. Here angular ordering restricts the region of allowed $z$ values in their branchings, but there are no matrix-element corrections. Once $m_{3}$ and $m_{4}$ are fixed, the kinematics of the $1 \rightarrow 3+4$ branching needs to be modified according to eq. (3). This is the reason why the evolution is always done for a pair of partons (whereof not both need branch further, however), and why the final kinematics of a branching is postponed to a later stage than the choice of $z$ value.

Several other aspects of the shower could be discussed, such as the choice of nonisotropic azimuthal angles to improve the coherence description and include gluon spin effects, the possibility also to emit photons, or the option to force some branchings in order to match higher-order matrix elements [14]. These are of less interest here and thus not covered. 


\subsection{The massless matrix element correction}

Let us now compare the parton-shower (PS) population of three-jet phase space with the matrix-element (ME) one, for the case of $\mathrm{e}^{+} \mathrm{e}^{-}$annihilation to massless quarks. With the conventional ME numbering $\mathrm{q}(1) \overline{\mathrm{q}}(2) \mathrm{g}(3)$, with $x_{j}=2 E_{j} / E_{\mathrm{CM}}$, the matrix element is of the form [15]

$$
\frac{1}{\sigma_{0}} \frac{\mathrm{d} \sigma_{\mathrm{ME}}}{\mathrm{d} x_{1} \mathrm{~d} x_{2}}=\frac{\alpha_{\mathrm{s}}}{2 \pi} C_{F} \frac{x_{1}^{2}+x_{2}^{2}}{\left(1-x_{1}\right)\left(1-x_{2}\right)},
$$

with the colour factor $C_{F}=4 / 3$. We have normalized to the lowest-order cross section $\sigma_{0}$, so that the expression can be interpreted as a probability distribution. For future reference, we will use the notation

$$
\frac{1}{\sigma_{0}} \frac{\mathrm{d} \sigma}{\mathrm{d} x_{1} \mathrm{~d} x_{2}}=\frac{\alpha_{\mathrm{s}}}{2 \pi} C_{F} \frac{N\left(x_{1}, x_{2}, r\right)}{\left(1-x_{1}\right)\left(1-x_{2}\right)},
$$

where $r=m_{\mathrm{q}} / E_{\mathrm{CM}}=m_{\overline{\mathrm{q}}} / E_{\mathrm{CM}}$. Thus $N_{\mathrm{ME}}\left(x_{1}, x_{2}, 0\right)=x_{1}^{2}+x_{2}^{2}$.

There are two shower histories that could give such a three-jet event. One is $\gamma^{*} / \mathrm{Z}^{0}(0) \rightarrow \mathrm{q}(i) \overline{\mathrm{q}}(2) \rightarrow \mathrm{q}(1) \overline{\mathrm{q}}(2) \mathrm{g}(3)$, i.e. with an intermediate $(i)$ quark branching $\mathrm{q}(i) \rightarrow \mathrm{q}(1) \mathrm{g}(3)$, illustrated in Fig. 1 $1 \mathrm{~b}$. This gives

$$
\begin{aligned}
Q^{2} & =m_{i}^{2}=\left(p_{0}-p_{2}\right)^{2}=\left(1-x_{2}\right) E_{\mathrm{CM}}^{2} \\
z & =\frac{p_{0} p_{1}}{p_{0} p_{i}}=\frac{E_{1}}{E_{i}}=\frac{x_{1}}{x_{1}+x_{3}}=\frac{x_{1}}{2-x_{2}}, \\
& \Rightarrow \frac{\mathrm{d} Q^{2}}{Q^{2}} \frac{\mathrm{d} z}{1-z}=\frac{\mathrm{d} x_{2}}{1-x_{2}} \frac{\mathrm{d} x_{1}}{x_{3}}
\end{aligned}
$$

The parton-shower probability for such a branching is

$$
\frac{\alpha_{\mathrm{s}}}{2 \pi} C_{F} \frac{1+z^{2}}{1-z} \mathrm{~d} z \frac{\mathrm{d} Q^{2}}{Q^{2}}=\frac{\alpha_{s}}{2 \pi} C_{F}\left[1+\left(\frac{x_{1}}{2-x_{2}}\right)^{2}\right] \frac{1-x_{1}}{x_{3}} \frac{\mathrm{d} x_{1} \mathrm{~d} x_{2}}{\left(1-x_{1}\right)\left(1-x_{2}\right)} .
$$

In the second shower history, the rôles of $\mathrm{q}$ and $\overline{\mathrm{q}}$ are interchanged, i.e. $x_{1} \leftrightarrow x_{2}$. This is the same set of Feynman graphs as in the matrix-element description, except that the shower does not include any interference between the two diagrams. The two shower expressions can therefore be added to give the overall shower population of the three-jet phase space, of the form in eq. (10) but with

$$
N_{\mathrm{PS}}\left(x_{1}, x_{2}, 0\right)=\frac{1-x_{1}}{x_{3}}\left(1+\left(\frac{x_{1}}{2-x_{2}}\right)^{2}\right)+\frac{1-x_{2}}{x_{3}}\left(1+\left(\frac{x_{2}}{2-x_{1}}\right)^{2}\right),
$$

In spite of $N_{\mathrm{PS}}$ being lengthier than $N_{\mathrm{ME}}$, it turns out that the two almost exactly agree over the whole phase space, but with the shower rate somewhat above, see e.g. Fig. 2. It is therefore straightforward and efficient to use the ratio

$$
R_{\mathrm{ME} / \mathrm{PS}}\left(x_{1}, x_{2}, 0\right)=\frac{\mathrm{d} \sigma_{\mathrm{ME}}}{\mathrm{d} \sigma_{\mathrm{PS}}}=\frac{N_{\mathrm{ME}}\left(x_{1}, x_{2}, 0\right)}{N_{\mathrm{PS}}\left(x_{1}, x_{2}, 0\right)}
$$

as an acceptance factor inside the shower evolution, in order to correct the first emission of the quark and antiquark to give a sum in agreement with the matrix element.

Clearly, the shower will contain further branchings that modify the simple result, e.g. by the emission both from the $\mathrm{q}$ and the $\overline{\mathrm{q}}$, but these effects are formally of $\mathcal{O}\left(\alpha_{\mathrm{s}}^{2}\right)$ and thus 


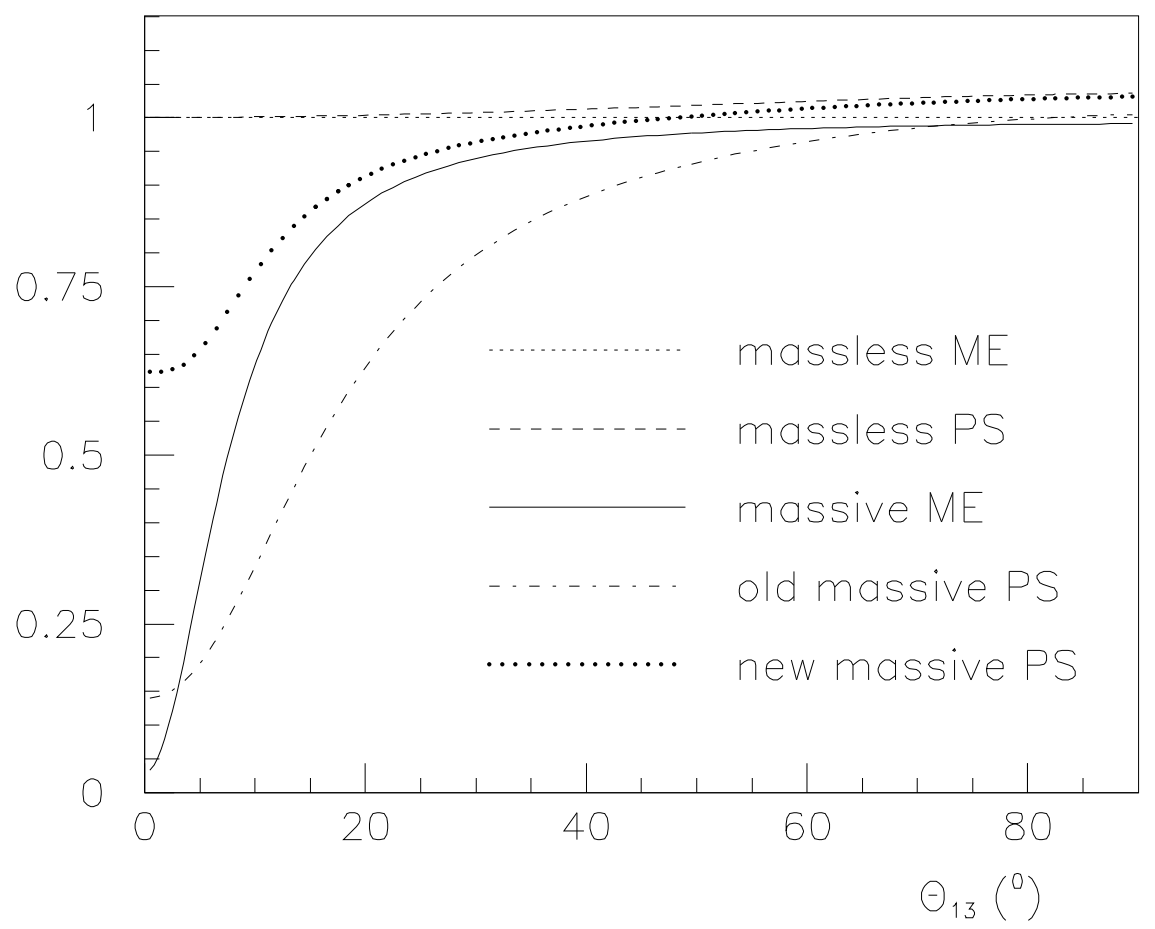

Figure 2: The gluon emission rate as a function of emission angle $\theta_{13}=\theta_{\mathrm{qg}}$, for a $10 \mathrm{GeV}$ gluon energy at $E_{\mathrm{CM}}=91 \mathrm{GeV}$, and with $m_{b}=4.8 \mathrm{GeV}$. All curves are normalized to the massless matrix-element expression, eq. (9), here thus represented by the small-dotted line at unity. Dashed: the massless shower before correction, $N_{\mathrm{PS}}\left(x_{1}, x_{2}, 0\right) / N_{\mathrm{ME}}\left(x_{1}, x_{2}, 0\right)=1 / R_{\mathrm{ME} / \mathrm{PS}}\left(x_{1}, x_{2}, 0\right)$. Full: the rate from massive matrix elements, $N_{\mathrm{ME}}\left(x_{1}, x_{2}, r\right) / N_{\mathrm{ME}}\left(x_{1}, x_{2}, 0\right)$. Dash-dotted: the rate from massive parton shower, $N_{\mathrm{PS}}\left(x_{1}, x_{2}, r\right) / N_{\mathrm{ME}}\left(x_{1}, x_{2}, 0\right)$. Large dots: the new rate from massive parton showers, $N_{\mathrm{PS}}^{\prime}\left(x_{1}, x_{2}, r\right) / N_{\mathrm{ME}}\left(x_{1}, x_{2}, 0\right)$.

beyond the accuracy we strive to match. One should also note that the shower modifies the distribution in three-jet phase space by the appearance of Sudakov form factors, and by using a running $\alpha_{\mathrm{s}}\left(p_{\perp}^{2}\right)$ rather than a fixed one. In both these respects, however, the shower should be an improvement over the fixed-order result.

\subsection{The massive matrix element correction}

The prescription of correcting the first branchings by the factor in eq. (16) was the original one, used up until Jetset 7.3, for massless and massive quarks alike, where the $x_{i}=$ $2 E_{i} / E_{\mathrm{CM}}$ variables were defined with masses included. In version 7.4 an intermediate 'improvement' was introduced, in that the massive matrix element expression was used, as given for a vector source like the $\gamma^{*}$ [16]:

$$
N_{\mathrm{ME}}\left(x_{1}, x_{2}, r\right)=x_{1}^{2}+x_{2}^{2}-4 r^{2} x_{3}-8 r^{4}-\left(2 r^{2}+4 r^{4}\right)\left(\frac{1-x_{2}}{1-x_{1}}+\frac{1-x_{1}}{1-x_{2}}\right) .
$$

The shower algorithm itself was not changed, nor the assumed shower weight, i.e. an acceptance factor $N_{\mathrm{ME}}\left(x_{1}, x_{2}, r\right) / N_{\mathrm{PS}}\left(x_{1}, x_{2}, 0\right)$ was applied for the first branching on either side. (The older behaviour remained as an option.)

The mass suppression in the matrix element is illustrated in Fig. 2. We remind that, in the soft-gluon limit, a spin-independent (and thus universal) eikonal expression holds 
[8]:

$$
\begin{aligned}
\frac{\mathrm{d} \sigma_{\mathrm{q} \overline{\mathrm{q}}}}{\sigma_{\mathrm{q} \overline{\mathrm{q}}}} & \propto(-1)\left(\frac{p_{1}}{p_{1} p_{3}}-\frac{p_{2}}{p_{2} p_{3}}\right)^{2} \frac{\mathrm{d}^{3} p_{3}}{E_{3}} \\
& =\left(\frac{2 p_{1} p_{2}}{\left(p_{1} p_{3}\right)\left(p_{2} p_{3}\right)}-\frac{m_{1}^{2}}{\left(p_{1} p_{3}\right)^{2}}-\frac{m_{2}^{2}}{\left(p_{2} p_{3}\right)^{2}}\right) E_{3} \mathrm{~d} E_{3} \mathrm{~d} \cos \theta_{13} .
\end{aligned}
$$

In the limit of small angles $\theta_{13}$ this gives a mass suppression factor

$$
\frac{\mathrm{d} \sigma\left(x_{3}, \theta_{13}, r\right)}{\mathrm{d} \sigma\left(x_{3}, \theta_{13}, 0\right)} \approx\left(\frac{\theta_{13}^{2}}{\theta_{13}^{2}+4 r^{2}}\right)^{2},
$$

i.e. the characteristic dead cone of opening angle approximately $2 r=m_{\mathrm{q}} / E_{\mathrm{q}}$. Note that the mapping between $\left(x_{1}, x_{2}\right)$ and $\left(x_{3}, \theta_{13}\right)$ depends on $r$, so eq. (19) is not quite the same as $N_{\mathrm{ME}}\left(x_{1}, x_{2}, r\right) / N_{\mathrm{ME}}\left(x_{1}, x_{2}, 0\right)$.

\subsection{The massive phase space correction in the shower}

More recently [5], the issue of masses in the shower was further studied, since the expression for $N_{\mathrm{PS}}\left(x_{1}, x_{2}, 0\right)$ had not been touched when $N_{\mathrm{ME}}$ was improved.

In the derivation of $N_{\mathrm{PS}}\left(x_{1}, x_{2}, r\right)$, one can start from the ansatz

$$
\begin{aligned}
& x_{2}=1-\frac{m_{i}^{2}-m_{\mathrm{q}}^{2}}{E_{\mathrm{CM}}^{2}}, \\
& x_{1}=\left(1+\frac{m_{i}^{2}-m_{\mathrm{q}}^{2}}{E_{\mathrm{CM}}^{2}}\right)\left(\left(1-k_{1}\right) z+k_{3}(1-z)\right), \\
& x_{3}=\left(1+\frac{m_{i}^{2}-m_{\mathrm{q}}^{2}}{E_{\mathrm{CM}}^{2}}\right)\left(\left(1-k_{3}\right)(1-z)+k_{1} z\right) .
\end{aligned}
$$

The quark mass enters both in the energy sharing between the intermediate quark $i$ and the antiquark 2, and in the correction procedure of eq. (3i) for the splitting of energy in the branching $q(i) \rightarrow q(1) g(3)$. The constraints $p_{1}^{2}=m_{\mathrm{q}}^{2}$ and $p_{3}^{2}=0$ give $k_{1}=0$ and $k_{3}=m_{\mathrm{q}}^{2} / m_{i}^{2}$. One then obtains

$$
\begin{aligned}
Q^{2} & =m_{i}^{2}=\left(1-x_{2}+r^{2}\right) E_{\mathrm{CM}}^{2}, \\
z & =\frac{1}{2-x_{2}}\left(x_{1}-r^{2} \frac{x_{3}}{1-x_{2}}\right) \\
& \Rightarrow \frac{\mathrm{d} Q^{2}}{Q^{2}} \frac{\mathrm{d} z}{1-z}=\frac{\mathrm{d} x_{2}}{1-x_{2}+r^{2}} \frac{\mathrm{d} x_{1}}{x_{3}} .
\end{aligned}
$$

This gives the answer

$$
\begin{aligned}
N_{\mathrm{PS}}\left(x_{1}, x_{2}, r\right) & =\frac{1-x_{1}}{x_{3}} \frac{1-x_{2}}{1-x_{2}+r^{2}}\left[1+\frac{1}{\left(2-x_{2}\right)^{2}}\left(x_{1}-r^{2} \frac{x_{3}}{1-x_{2}}\right)^{2}\right] \\
& +\left\{x_{1} \leftrightarrow x_{2}\right\},
\end{aligned}
$$

where the second term comes from the graph where the antiquark radiates.

The mass effects go in the 'right' direction, $N_{\mathrm{PS}}\left(x_{1}, x_{2}, r\right)<N_{\mathrm{PS}}\left(x_{1}, x_{2}, 0\right)$, but actually so much so that $N_{\mathrm{PS}}\left(x_{1}, x_{2}, r\right)<N_{\mathrm{ME}}\left(x_{1}, x_{2}, r\right)$ in major regions of phase space. This is 
illustrated in Fig. 2. Very crudely, one could say that the massive shower exaggerates the angle of the dead cone by about a factor of two, in this rather typical example. There is no dead cone as such built in, however, but a rather more coincidental mass suppression mainly generated by the factor $\left(1-x_{2}\right) /\left(1-x_{2}+r^{2}\right)$.

Thus the amount of gluon emission off massive quarks is underestimated already in the original prescription, where masses entered in the kinematics but not in the ME/PS correction factor. When the intermediate 'correction' ratio $N_{\mathrm{ME}}\left(x_{1}, x_{2}, r\right) / N_{\mathrm{PS}}\left(x_{1}, x_{2}, 0\right)$ is applied, the net result is a distribution even more off from the correct one, by a factor $N_{\mathrm{PS}}\left(x_{1}, x_{2}, r\right) / N_{\mathrm{PS}}\left(x_{1}, x_{2}, 0\right)$. Thus it would have been better not to introduce the mass correction in JETSET 7.4.

Based on the results above, one can now instead use the correct ME/PS factor $N_{\mathrm{ME}}\left(x_{1}, x_{2}, r\right) / N_{\mathrm{PS}}\left(x_{1}, x_{2}, r\right)$. A technical problem is that this ratio can exceed unity, in the example of Fig. 2 by up to almost a factor of two. This could be solved e.g. by enhancing the raw rate of emissions by this factor. However, another trick was applied, based on the facts that the shift of eqs. (3) and (20) implies that smaller-energy gluons would be allowed for a massive quark than a massless one, and additionally that the accessible $z$ range is overestimated in the original ansatz. Therefore, without any (noticeable) loss of phase space, $z$ can be rescaled to a $z^{\prime}$ according to

$$
\left(1-z^{\prime}\right)=(1-z)^{k}, \quad \text { with } \quad k=\frac{\ln \left(r^{2}\right)}{\ln \left(Q_{0}^{2} / E_{\mathrm{CM}}^{2}\right)}<1 .
$$

The ME/PS correction factor then has to be compensated by $k$, and thereby comes below unity almost everywhere - the remaining weighting errors are too small to be relevant.

This procedure, default since PyтнIA version 6.130, improves the shower description of mass effects in the amount of three-jet events [0]. Mass effects are only included correctly for the first branching of the $q$ and $\bar{q}$ in the shower, however. Subsequent emissions involve no correction procedure. Instead the dead cone effect is exaggerated, similarly to what was shown in Fig. 2. Furthermore, even for the first branchings, only the possibility of a vector source decaying to two identical-mass quarks is included, while the $\mathrm{Z}^{0}$ actually is a mixture of vector and axial vector, and e.g. the $\mathrm{W}^{ \pm}$would decay to two unequal-mass quarks. We will therefore next try to develop a more powerful and general approach.

\section{A New Approach}

One of the advantages of the Monte Carlo approach is that, so long as some upper estimate can be found that allows simple generation, rejection down to a more complex expression is straightforward. In particular, for an evolution in some variable $Q$ with a (Sudakov) form factor built up from the no-emission probability, the 'veto algorithm' [6] can be used, in which the initial overestimate of the emission rate is compensated by the possibility of a rejection, with a continued evolution downwards in $Q^{2}$ from the rejected value onwards. As we have seen, the current set of $Q^{2}$ and $z$ variables is not so convenient, since the emission rate off massive quarks is an underestimate of the correct rate, and therefore the standard procedure does not work except after some extra tricks. We will therefore pick another set of variables, preferably such that they reduce to the old ones in the massless limit. Several approaches could have been taken, but here we have chosen the minimal one of retaining the $z$ definition and thereby the existing kinematics machinery. As we will show, a modification for the branching $a \rightarrow b c$ from $Q^{2}=m_{a}^{2}$ to $Q^{2}=m_{a}^{2}-\left(m_{a}^{2}\right)_{\text {on-shell }}$ is enough to do the job. 


\subsection{The choice of shower variables}

Again consider $\mathrm{e}^{+} \mathrm{e}^{-} \rightarrow \gamma^{*} / \mathrm{Z}^{0} \rightarrow \mathrm{q}(1) \overline{\mathrm{q}}(2) \mathrm{g}(3)$, and the formulae of eq. (20), but now assume $Q^{2}=m_{i}^{2}-m_{\mathrm{q}}^{2}=m_{i}^{2}-m_{1}^{2}$ for the $\mathrm{q}(i) \rightarrow \mathrm{q}(1) \mathrm{g}(3)$ branching. That corresponds to $1 / Q^{2}$ being the propagator of the off-shell parton 1 . Then eq. (22) is unchanged while

$$
\begin{aligned}
Q^{2} & =m_{i}^{2}-m_{1}^{2}=\left(1-x_{2}\right) E_{\mathrm{CM}}^{2} \\
& \Rightarrow \frac{\mathrm{d} Q^{2}}{Q^{2}} \frac{\mathrm{d} z}{1-z}=\frac{\mathrm{d} x_{2}}{1-x_{2}} \frac{\mathrm{d} x_{1}}{x_{3}} .
\end{aligned}
$$

So, with this simple trick, we have recovered the Jacobian of the massless case, eq. (13). This means that there is little mass suppression left in the shower evolution proper:

$$
\begin{aligned}
N_{\mathrm{PS}}^{\prime}\left(x_{1}, x_{2}, r\right) & =\frac{1-x_{1}}{x_{3}}\left[1+\frac{1}{\left(2-x_{2}\right)^{2}}\left(x_{1}-r^{2} \frac{x_{3}}{1-x_{2}}\right)^{2}\right] \\
& +\left\{x_{1} \leftrightarrow x_{2}\right\}
\end{aligned}
$$

as can be seen in Fig. 2. In particular, note that $N_{\mathrm{PS}}^{\prime}\left(x_{1}, x_{2}, r\right)$ reduces to $N_{\mathrm{PS}}\left(x_{1}, x_{2}, 0\right)$, eq. (14), in the soft limit $x_{3} \rightarrow 0$. Therefore a matrix-element correction now is even more required, but also simpler to implement, to bring down the rate to a reasonable level.

So far, we have assumed decay to two equal-mass quarks, like in $\mathrm{Z}^{0}$ decay. For many of the examples to be discussed, like $\mathrm{W}^{ \pm}$or $\mathrm{t}$ decay, the two decay products have unequal masses. It is also convenient to allow slightly unequal masses for particles with nonnegligible widths, e.g. in $\mathrm{e}^{+} \mathrm{e}^{-} \rightarrow \mathrm{t} \overline{\mathrm{t}}$. We will therefore generalize to the case with $r_{1}=m_{1} / E_{\mathrm{CM}}=m_{\mathrm{q}} / E_{\mathrm{CM}} \neq r_{2}=m_{2} / E_{\mathrm{CM}}=m_{\overline{\mathrm{q}}} / E_{\mathrm{CM}}$. The range of kinematically allowed $x_{i}$ values is then $2 r_{1} \leq x_{1} \leq 1+r_{1}^{2}-r_{2}^{2}, 2 r_{2} \leq x_{2} \leq 1+r_{2}^{2}-r_{1}^{2}, 0 \leq x_{3} \leq 1-\left(r_{1}+r_{2}\right)^{2}$, with the joint condition that

$$
\left(2-2 x_{1}-2 x_{2}+x_{1} x_{2}+2 r_{1}^{2}+2 r_{2}^{2}\right)^{2} \leq\left(x_{1}^{2}-4 r_{1}^{2}\right)\left(x_{2}^{2}-4 r_{2}^{2}\right) .
$$

The ansatz of eq. (20) is modified by $m_{\mathrm{q}} \rightarrow m_{2}$ while $k_{3} \rightarrow m_{1}^{2} / m_{i}^{2}$. Furthermore

$$
\begin{aligned}
Q^{2} & =m_{i}^{2}-m_{1}^{2}=\left(p_{0}-p_{2}\right)^{2}-m_{1}^{2}=\left(1+r_{2}^{2}-r_{1}^{2}-x_{2}\right) E_{\mathrm{CM}}^{2} \\
z & =\frac{1}{2-x_{2}}\left(x_{1}-r_{1}^{2} \frac{x_{3}}{1+r_{2}^{2}-r_{1}^{2}-x_{2}}\right) \\
& \Rightarrow \frac{\mathrm{d} Q^{2}}{Q^{2}} \frac{\mathrm{d} z}{1-z}=\frac{\mathrm{d} x_{2}}{1+r_{2}^{2}-r_{1}^{2}-x_{2}} \frac{\mathrm{d} x_{1}}{x_{3}}
\end{aligned}
$$

When a colourless particle decays to two colour triplets, the two possible one-gluonemission shower histories then add up to give

$$
\frac{1}{\sigma_{0}} \frac{\mathrm{d} \sigma_{\mathrm{PS}}}{\mathrm{d} x_{1} \mathrm{~d} x_{2}}=\frac{\alpha_{\mathrm{s}}}{2 \pi} C_{F}\left\{\frac{1+z_{1}^{2}}{x_{3}\left(1+r_{2}^{2}-r_{1}^{2}-x_{2}\right)}+\frac{1+z_{2}^{2}}{x_{3}\left(1+r_{1}^{2}-r_{2}^{2}-x_{1}\right)}\right\} .
$$

Here $z_{1}$ is given by eq. (31) and $z_{2}$ is obtained by exchanging $1 \leftrightarrow 2$. Since the numerators $1 \leq 1+z_{i}^{2} \leq 2$, their exact form is not a main concern for the qualitative discussions. As it turns out, the Monte Carlo procedure is simplified if the shower is generated with a numerator 2 instead of $1+z_{i}^{2}$, so we will make this replacement in the following, with the matrix element correction procedure compensating this overestimate. 


\subsection{The matrix element correction}

Next, one should try to relate this to the structure of the matrix elements under similar conditions. We may expect graphs where either of the partons can radiate a gluon and therefore can give a propagator $1 / Q_{1}^{2}=1 /\left(m_{13}^{2}-m_{1}^{2}\right)$, cf. eq. (30), or $1 / Q_{2}^{2}=1 /\left(m_{23}^{2}-m_{2}^{2}\right)$, depending on which side radiates. (Diagrams with a four-boson vertex, such as $\gamma^{*} / \mathrm{Z}^{*} \rightarrow$ $\tilde{\tilde{t}} \overline{\mathrm{t} g}$, are not singular and therefore do not affect the discussion.) After summing and squaring, the cross section then should have the form

$$
\begin{aligned}
\frac{1}{\sigma_{0}} \frac{\mathrm{d} \sigma_{\mathrm{ME}}}{\mathrm{d} x_{1} \mathrm{~d} x_{2}}= & \frac{\alpha_{\mathrm{s}}}{2 \pi} C_{F}\left\{\frac{E_{\mathrm{CM}}^{4} A\left(x_{1}, x_{2}\right)}{\left(m_{13}^{2}-m_{1}^{2}\right)^{2}}+\frac{E_{\mathrm{CM}}^{4} B\left(x_{1}, x_{2}\right)}{\left(m_{23}^{2}-m_{2}^{2}\right)^{2}}+\frac{E_{\mathrm{CM}}^{4} C\left(x_{1}, x_{2}\right)}{\left(m_{13}^{2}-m_{1}^{2}\right)\left(m_{23}^{2}-m_{2}^{2}\right)}\right\} \\
= & \frac{\alpha_{\mathrm{s}}}{2 \pi} C_{F}\left\{\frac{A\left(x_{1}, x_{2}\right)}{\left(1+r_{2}^{2}-r_{1}^{2}-x_{2}\right)^{2}}+\frac{B\left(x_{1}, x_{2}\right)}{\left(1+r_{1}^{2}-r_{2}^{2}-x_{1}\right)^{2}}\right. \\
& \left.+\frac{C\left(x_{1}, x_{2}\right)}{\left(1+r_{2}^{2}-r_{1}^{2}-x_{2}\right)\left(1+r_{1}^{2}-r_{2}^{2}-x_{1}\right)}\right\} .
\end{aligned}
$$

The individual functions $A, B$ and $C$ depend on the gauge choice, but the total cross section of course is gauge-independent. It also has some general features, which can be seen in the soft-gluon limit expression, eq. (18). Here the interference term is giving a positive expression and the seemingly quadratically-divergent terms are negative and drive the cross section to zero in the collinear limit, the dead cone. In order to have an upper estimate of the cross section, for Monte Carlo applications, it is thus enough to have the singularity structure of the interference term modelled, and no need to worry about quadratic divergences.

As before, we could now compare the total shower and total matrix element rates, and define a corrective factor between the two. To simplify some of the continued studies, however, we have adopted an alternative approach. Instead of adding the two shower histories to compare with the full matrix element, one can split the matrix element in two, such that each part can be compared with only one shower history. Such a subdivision of course is arbitrary, but should still be sensible. A gluon emitted close to the $p_{1}$ direction should predominantly be emitted by parton 1 , and vice versa. A suitable such subdivision is in the proportions given by the propagators, $1 / Q_{1}^{2}: 1 / Q_{2}^{2}$, which also is the proportions between the two shower histories (in the $1+z^{2} \rightarrow 2$ approximation). For massless partons, and going to the soft-gluon limit, this means a probability $\left(1+\cos \theta_{13}\right) / 2=\left(1-\cos \theta_{23}\right) / 2$ for emission off parton 1 and the rest for emission off parton 2 .

In the description of the gluon emission rate off parton 1, a matrix element fraction

$$
W_{\mathrm{ME}, 1}\left(x_{1}, x_{2}\right)=\frac{Q_{2}^{2}}{Q_{1}^{2}+Q_{2}^{2}} \frac{1}{\sigma_{0}} \frac{\mathrm{d} \sigma_{\mathrm{ME}}}{\mathrm{d} x_{1} \mathrm{~d} x_{2}}=\frac{1+r_{1}^{2}-r_{2}^{2}-x_{1}}{x_{3}} \frac{1}{\sigma_{0}} \frac{\mathrm{d} \sigma_{\mathrm{ME}}}{\mathrm{d} x_{1} \mathrm{~d} x_{2}}
$$

should be compared with the first half of the total parton shower expression in eq. (33),

$$
W_{\mathrm{PS}, 1}\left(x_{1}, x_{2}\right)=\frac{\alpha_{\mathrm{s}}}{2 \pi} C_{F} \frac{2}{x_{3}\left(1+r_{2}^{2}-r_{1}^{2}-x_{2}\right)} .
$$

The ME/PS correction factor then becomes

$$
\begin{aligned}
R_{1}\left(x_{1}, x_{2}\right) & =\frac{W_{\mathrm{ME}, 1}\left(x_{1}, x_{2}\right)}{W_{\mathrm{PS}, 1}\left(x_{1}, x_{2}\right)} \\
& =\frac{\left(1+r_{2}^{2}-r_{1}^{2}-x_{2}\right)\left(1+r_{1}^{2}-r_{2}^{2}-x_{1}\right)}{2}\left(\frac{\alpha_{\mathrm{s}}}{2 \pi} C_{F}\right)^{-1} \frac{1}{\sigma_{0}} \frac{\mathrm{d} \sigma_{\mathrm{ME}}}{\mathrm{d} x_{1} \mathrm{~d} x_{2}},
\end{aligned}
$$


which reduces to $R_{1}\left(x_{1}, x_{2}\right)=N_{\mathrm{ME}}\left(x_{1}, x_{2}, r\right) / 2$ for $r_{1}=r_{2}=r$.

The intention is that $R_{1}\left(x_{1}, x_{2}\right)$ should be finite and well-behaved over all of phase space, with one factor of each divergence now multiplied on to the matrix element expression. We will illustrate the behaviour later on, but the key observation is that, for all the matrix elements we will study, searches over the full mass parameter plane and phase space have failed to find any point where the ratio is above unity. $R_{1}$ therefore serves well as simple rejection factor. (Without the replacement $1+z^{2} \rightarrow 2$ in the shower description one would find $R_{1}\left(x_{1}, x_{2}\right)>1$, and some extra precautions would be necessary.) The correction factor $R_{2}$ for the other shower history looks the same. Put another way, the relative probability for a gluon to be emitted by parton 1 or by parton 2 is unchanged by the matrix element correction procedure.

The above procedure works not only for quarks, but also e.g. for squarks. The numerator of the splitting kernel would have been $2 z$ rather than $1+z^{2}$ [18], but both are equally well approximated from above by 2 . For a gluino, the colour charge is $N_{C}$ rather than $C_{F}$, so the assumed shower emission rate has to be scaled up by a factor $N_{C} / C_{F}=9 / 4$ (also for the recoiling colour triplet parton, since the separation of radiation is not perfect), with no other change required.

We will also encounter processes where the decaying particle carries colour, like in $t \rightarrow$ $\mathrm{bW}^{+}$. Then gluon emission off this particle has to be considered, i.e. graphs like $\mathrm{t}(0) \rightarrow$ $\mathrm{t}(i) \mathrm{g}(3) \rightarrow \mathrm{b}(1) \mathrm{W}^{+}(2) \mathrm{g}(3)$, proceeding via an intermediate off-shell top. Neglecting the top width, this introduces a new kind of inverse propagators

$$
\left|Q_{0}^{2}\right|=m_{0}^{2}-m_{i}^{2}=m_{123}^{2}-m_{12}^{2}=2\left(p_{1}+p_{2}\right) p_{3}=x_{3} E_{\mathrm{CM}}^{2} .
$$

The leading term of the matrix element, proportional to $1 /\left(Q_{0}^{2} Q_{1}^{2}\right)$, will therefore have the same singularity structure as the shower rate in eq. (36). In a shower description, where only the $\mathrm{b}$ is allowed to radiate, there is thus no problem in principle of letting that radiation account for the full emission pattern of the matrix elements, i.e.

$$
\begin{aligned}
R_{1}\left(x_{1}, x_{2}\right) & =\frac{W_{\mathrm{ME}}\left(x_{1}, x_{2}\right)}{W_{\mathrm{PS}, 1}\left(x_{1}, x_{2}\right)} \\
& =\frac{x_{3}\left(1+r_{2}^{2}-r_{1}^{2}-x_{2}\right)}{2}\left(\frac{\alpha_{\mathrm{s}}}{2 \pi} C_{F}\right)^{-1} \frac{1}{\sigma_{0}} \frac{\mathrm{d} \sigma_{\mathrm{ME}}}{\mathrm{d} x_{1} \mathrm{~d} x_{2}} .
\end{aligned}
$$

Since also here the shower rate turns out to exceed the matrix element one, a simple rejection approach should work well.

\subsection{Subsequent branchings}

So far, we have considered matrix elements as providing the probability of exclusive three-jet events. An alternative interpretation, however, would be in terms of an inclusive density of gluon emissions, with the possibility of several such per event. This interpretation works well in the soft-gluon limit, while the emission of a hard gluon reduces the phase space for subsequent emissions and thus ruins the picture of independent emissions. Furthermore, the possibility for gluons to branch in their turn leads to the need to include coherence effects [17] that constrain allowed emissions. Nevertheless, the matrix elements can be used to extract important information, not only on the emission rate of hard gluons, but also on that of soft and collinear ones.

Historically, different approaches have therefore been taken. In the JETSET/PYTHIA procedure used until now, only the first branching is corrected by matrix-element information. Subsequent emissions involve no corrections, but only the process-independent 
splitting kernels, and therefore give the wrong emission rate off heavy quarks that has already been noted. In the HERWIG routine, a correction is performed for every emission that is the hardest so far [12] - whereas JETSET emissions tend to be ordered in hardness, this is less likely in HERWIG, so the distinction is then relevant. ARIADNE, finally, imposes a correction at all steps of the cascade.

Since our new algorithm does not have the correct dead-cone behaviour built in from the onset, it is clear that some correction procedure will be required. Rather than imposing the process-independent collinear behaviour, we have chosen to base ourselves on the matrix elements also in this region. This offers a smooth interpolation between the hard process-specific and the soft collinear universal behaviours, and sidesteps the issue of which emissions are to be considered the hardest so far [12]. Thus the shower rate will be corrected to the matrix element one at every step of the shower off the original partons. The gluon cascading $\mathrm{g} \rightarrow \mathrm{gg}$ is unaffected, since there are no gluon-mass effects or matrix elements to be considered here. Light quarks (in $\gamma^{*} / \mathrm{Z}^{0}$ decay) are also essentially unaffected, since there the shower matches so well with the matrix elements anyway.

The kinematics of the cascade changes in each emission, as the energy of the radiating parton is reduced by the previous emissions. The mapping of an emission on to the matrix element variables is thereby not unique. However, from the dead cone formula, eq. (19), we see that the emission angle and the mass-to-energy ratio of the emitting quark should be represented faithfully in the choice of matching matrix element variables. For the branchings subsequent to the first one, say $3 \rightarrow 7+8$ in Fig. 1 1 a, the mother energy $E_{3}$ is fixed. The choice of a $Q^{2}=m_{3}^{2}-m_{7}^{2}$ and a $z$ of the branching maps onto four-momenta $p_{7}$ and $p_{8}$ as described in eqs. (3) $-(17)$, with $p_{7}^{2}=m_{\mathrm{q}}^{2}>0$ and $p_{8}^{2}=0$. In order to make contact with the matrix element variables, now construct a hypothetical recoiling parton $2^{\prime}$, such that $\mathbf{p}_{2}^{\prime}=-\mathbf{p}_{3}$ and ${p_{2}^{\prime}}^{2}=m_{\overline{\mathrm{q}}}^{2}$. The CM energy of this reduced system is then given by

$$
E_{\mathrm{CM}}^{\prime}=E_{3}+E_{2}^{\prime}=E_{3}+\sqrt{\mathbf{p}_{3}^{2}+m_{\overline{\mathrm{q}}}^{2}}=E_{3}+\sqrt{E_{3}^{2}-Q^{2}-m_{\mathrm{q}}^{2}+m_{\overline{\mathrm{q}}}^{2}} .
$$

Now matrix element and parton shower weights can be evaluated for $x_{1}^{\prime}=2 E_{7} / E_{\mathrm{CM}}^{\prime}$, $x_{2}^{\prime}=2 E_{2}^{\prime} / E_{\mathrm{CM}}^{\prime}, r_{1}^{\prime}=m_{\mathrm{q}} / E_{\mathrm{CM}}^{\prime}$ and $r_{2}^{\prime}=m_{\overline{\mathrm{q}}} / E_{\mathrm{CM}}^{\prime}$. Note that, in the limit $E_{4} \rightarrow 0$, also $E_{\mathrm{CM}}^{\prime} \rightarrow E_{\mathrm{CM}}$, and the ordinary matrix elements are recovered.

The above procedure is not unique. As an alternative, one could retain the original $E_{\mathrm{CM}}$, but construct an off-shell $p_{2}^{\prime}$ to carry all the energy and momentum of the system except for $p_{3}=p_{7}+p_{8}$, i.e. $p_{2}^{\prime}=p_{2}+p_{4}$ in the case of Fig. 1 1 a. This gives equivalent results so long as the gluon energy already emitted is not too large, and else gives a somewhat lower rate of wide-angle emission, reflecting that the parton $2^{\prime}$ then is assigned such a large mass that it radiates less. The overall picture therefore is less appealing, even if results in practical applications are almost equivalent.

In this article, emphasis is put on the gluon emission off the primary quarks (or other primary particles). The subsequent branchings $g \rightarrow$ gg have not been affected by the mass considerations, so are not discussed here. The rate of $g \rightarrow q \bar{q}$ branchings is another topic of some interest, given that LEP results do not quite agree with predictions [5]. This issue is further discussed in Sect. 6.

However, given that such a secondary $c \bar{c}$ or $b \bar{b}$ pair has been produced, the possibility of further gluon emission off this pair should be considered, even if most pairs are produced at such a low mass that the phase space left for further radiation is limited. In order to provide a sensible behaviour in the collinear region, again matrix-element input is applied, calculated for the decay of a colour octet source. (Process 66 of Table 1, so with the wrong 
spin of the source, but correct for the radiating parton, which is the main point.) To first approximation, this means that radiation occurs independently off the $q$ and $\bar{q}$; see the discussion on radiation patterns below. The kinematics for the matrix element corrections is set up about as described above, i.e. by mapping onto a reduced system at rest with preserved energy for the radiating parton. A simplification is that here the recoiling parton always has the same mass as the radiating one.

\subsection{Additional issues}

In the older shower algorithm, the decay products of a branching $a \rightarrow b c$ were assumed massless until assigned a mass by some subsequent step. This meant that the $p_{\perp}^{2}$ of a branching, eq. (7) with $\beta_{a}=1$ as a first simplification, was simplified further to $p_{\perp}^{2} \approx z(1-$ z) $m_{a}^{2}$ when used as argument in $\alpha_{\mathrm{s}}\left(p_{\perp}^{2}\right)$. In a branching $\mathrm{q} \rightarrow \mathrm{qg}$, where the $\mathrm{q}$ is assumed to have a non-vanishing rest mass, one would instead obtain $p_{\perp}^{2} \approx z(1-z) m_{a}^{2}\left(1-m_{b}^{2} / m_{a}^{2}\right)^{2}$. (This follows from eq. (7) or alternatively from the rescaling of $x_{3}$ in eq. (20).) A smaller $p_{\perp}^{2}$ estimate implies a larger $\alpha_{\mathrm{s}}$ and emission rate for a given kinematical configuration, but also that more phase space is cut out by the requirement $p_{\perp}^{2}>Q_{0}^{2} / 4$, where $Q_{0}$ is the soft cut-off scale of the cascade. The net result of such a change is therefore not obvious, and we will study it later on.

Also the calculation of the approximate opening angle of a branching $a \rightarrow b c$, eq. (8), would be affected by the same considerations. The massive parton energy $E_{b}$ is increased at the expense of $E_{c}$, as given by eq. (20). Since the common $p_{\perp}$ is also decreased, the ratio $p_{\perp} / E_{c}$ is preserved, while $p_{\perp} / E_{b}$ is decreased. The net result is a decrease of the opening angle by a factor $\left(1+\left(m_{b}^{2} / m_{a}^{2}\right)(1-z) / z\right)^{-1}$. As another option, we will then consider the consequences of such a decrease in the decay opening angle, without any change of the production angle, as a way of minimally relaxing the angular ordering condition off massive quarks.

While our choice of $Q^{2} \approx m^{2}$ variable has significant advantages for the matching to matrix-element expressions, it does not offer as neat an implementation of coherence effects as the angular variable of HERWIG or the transverse momentum one of ARIADNE. Without any further constraints, the amount of radiation is overestimated. Therefore, by default, angular ordering of emissions is imposed as a further constraint. This, on the other hand, tends to restrict emissions somewhat too much. The bulk of these ambiguities affect rather soft gluons, which do not give rise to separate jets. For the precision studies in Section 5.1, however, also small effects could be of interest. We have therefore introduced a new "intermediate" coherence option, as compared to the minimal modification above, wherein no angular constraint is imposed on emissions off the primary q $\bar{q}$ pair. These emissions thus are ordered only in mass. Angular ordering is still imposed in the cascades initiated by the gluons emitted off the primary quarks. In particular each such cascade is restricted to a cone given by the emission angle of the initiating gluon. In this option, generic event properties are only slightly changed compared with the default procedure.

So far, we have mainly considered configurations where a colour singlet decays to stable particles, e.g. $\gamma^{*} / \mathrm{Z}^{0} \rightarrow \mathrm{b} \overline{\mathrm{b}}$. Another class of events involve sequential decays of coloured objects. The obvious example would be $\gamma^{*} / \mathrm{Z}^{*} \rightarrow \mathrm{t} \overline{\mathrm{t}} \rightarrow \mathrm{bW} \mathrm{W}^{+} \overline{\mathrm{b}} \mathrm{W}^{-}$. Even leaving aside the continued fate of the W's, e.g. assuming they decay leptonically, the event now contains four colour charges that may radiate. In the limit $\Gamma_{t} \rightarrow 0$, the radiation in the top production stage $\gamma^{*} / \mathrm{Z}^{*} \rightarrow \mathrm{t} \overline{\mathrm{t}}$ decouples completely from that in the top decays. For a finite $\Gamma_{\mathrm{t}}$, gluons with energies below or around this scale can receive contributions from 


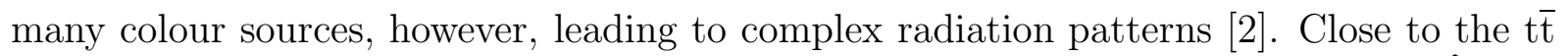
threshold, the main sources are the respective top decays, described by dipoles t $\widehat{b}$ and $\widehat{\bar{t} \bar{b}}$, and radiation off the $\widehat{b \bar{b}}$ dipole created after the $t$ and $\bar{t}$ have decayed. Gluons with energies above (below) $\Gamma_{\mathrm{t}}$ predominantly feel the former (latter) dipoles. The radiation pattern can be written as

$$
\frac{E_{\mathrm{g}}^{2}}{\Gamma_{\mathrm{t}}^{2}+E_{\mathrm{g}}^{2}}(\widehat{\mathrm{tb}}+\widehat{\overline{\mathrm{tb}}})+\frac{\Gamma_{\mathrm{t}}^{2}}{\Gamma_{\mathrm{t}}^{2}+E_{\mathrm{g}}^{2}} \widehat{\mathrm{b} \overline{\mathrm{b}}} .
$$

Thus the reduced radiation induced by the top finite lifetime is compensated by the radiation from new sources that would not have been present for a long-lived top. The $\widehat{\mathrm{b} \overline{\mathrm{b}}}$ dipole introduces a dependence on the opening angle between the $\mathrm{b}$ and the $\overline{\mathrm{b}}$ that is not there in the separate top decays, so the compensation is not complete. On the perturbative level, this gives a dipole effect [19] that could be observed at low momenta. However, even in the approximation of allowing radiation only within each top quark decay separately, the string fragmentation picture [1] would imply that a nonperturbative colour string should be stretched between the $\mathrm{b}$ and $\overline{\mathrm{b}}$ (or their respective cascades), and this would introduce a string effect [20] of almost equal character and magnitude [21]. Thus only very careful studies, e.g. for high-precision measurements of the top mass, would be sensitive to the detailed nature of the soft-gluon emission source. The critical transition is the one between a top long-lived enough to produce top hadrons and one decaying too rapidly for that, where the hadronic final state does change character.

The shower algorithm contains options that allows it to be run, either with soft or with hard emission dampened according to the respective factors in eq. (41). However, currently the PYTHIA program contains no machinery to detect when a description of this kind is required, nor a prescription how to combine all possible sources of radiation. This is obviously an interesting task for the future, but one that will be required primarily for particles with a width significantly larger than that of the top.

The normal generation sequence therefore contains a set of separated showers. For instance, in the top example above, the $\gamma^{*} / \mathrm{Z}^{*} \rightarrow \mathrm{t} \overline{\mathrm{t}}$ induces a first cascade, whereby the $\mathrm{t}$ and $\overline{\mathrm{t}}$ shower down to the mass shell. Thereafter follows the respective top decay, $\mathrm{t} \rightarrow \mathrm{bW}^{+}$and $\overline{\mathrm{t}} \rightarrow \overline{\mathrm{b}} \mathrm{W}^{-}$, and the separate radiation in those decays. At some yet later stage, the two W's may decay to quarks that again radiate. Anytime a colour singlet is exchanged, like the W's above, there is a clean separation into disjoint QCD subsystems, while the exchange of a coloured state like the top will hook up separately showering systems to the strings that later will produce the observable hadrons. Interconnection effects [22] could complicate this picture, but so far the evidence is that any such effects would be small.

In hadronic collisions, more complex processes would occur, and also initial-state QCD radiation has to be considered as a potential source of further interference effects. In this article we will not address these additional complications, but defer that for some future study. Currently such interference is almost completely neglected, except for some angular restrictions 23.

Even if $\Gamma_{\mathrm{t}}$ is neglected in the showering, for the sake of providing a unique separation of radiation before and after the top propagator, this does not mean that all top quarks have to have the same mass. Instead, resonance masses are chosen according to the relevant Breit-Wigners, convoluted with the respective cross section formulae. In particular, this means that the $t$ and $\bar{t}$ masses of an event would be unequal. The shower algorithm 
described above then operates on these event-by-event masses, without any reference to their nominal on-shell equivalents.

The hadronization of a partonic configuration, obtained by the chain of decays and showers outlined above, is described by the Lund string model [1]. All coloured partons belong to strings, stretched from a quark endpoint via a number of intermediate gluons to an antiquark one. (Also diquark endpoints and closed gluon loops can be considered, but are of no relevance in this article.) Normally each such string would have a reasonably large invariant mass, enough to produce several hadrons. However, occasionally a string could come to have a small mass, e.g. by the splitting of a string in two by shower branchings $g \rightarrow q \bar{q}$. Then a special treatment may be required for one- or two-hadron decays. While the normal string algorithm has remained essentially unchanged over a number of years, this low-mass 'cluster' treatment has recently been improved [24]. The direct consequences for the topics studied here are minimal, except that quark mass values have now been optimized, especially to describe charm asymmetries in fixed-target experiments. The current default values thus are $m_{\mathrm{u}}=m_{\mathrm{d}}=0.33 \mathrm{GeV}, m_{\mathrm{s}}=0.5 \mathrm{GeV}$, $m_{\mathrm{c}}=1.5 \mathrm{GeV}$ and $m_{\mathrm{b}}=4.8 \mathrm{GeV}$.

\section{Matrix elements}

As input and starting point for our shower studies, we will need the matrix elements for the processes of interest. In this article this essentially means the two-body decay of a particle, with associated gluon radiation. Some of the formulae are available in the literature, but most are not, or at least not easily found. We have therefore calculated a number of processes. This also gives us a chance to test the degree of universality of the radiation patterns in channels of different colour and spin structure, but with the same masses. These results are interesting in their own right.

\subsection{Calculations}

A number of matrix elements have been calculated, using COMPHEP [25] for the actual calculation, including an extension package for Supersymmetric processes [26], and Mathematica [27] for subsequent simplification of the expressions. The list of lowestorder (LO) processes is given in Table 1. The LO expression gives the two-body decay rate of a particle, i.e. $a \rightarrow b c$, and the matching first-order (FO) one the same decay with an additional gluon in the final state, $a \rightarrow b c g$.

While the matrix element calculations in this section have been performed from scratch, some checks are based on results in the literature. These include: $V \rightarrow \mathrm{q} \overline{\mathrm{q}}$ for $m_{\mathrm{q}}=m_{\overline{\mathrm{q}}}$ [16], $\mathrm{H}^{0} \rightarrow \mathrm{q} \overline{\mathrm{q}}$ for $m_{\mathrm{q}}=m_{\overline{\mathrm{q}}}$ [28], $V \rightarrow \tilde{\mathrm{q}} \overline{\tilde{\mathrm{q}}}$ for $m_{\tilde{\mathrm{q}}}=m_{\overline{\tilde{\mathrm{q}}}}$ [18, and $\mathrm{t} \rightarrow \mathrm{bW}^{+}$ for $m_{\mathrm{b}}=0$ [13]. No doubt, many more are available, without our knowledge.

The process selection is based on the particle content of the Minimal Supersymmetric Standard Model (MSSM), i.e. includes squarks $\tilde{\mathrm{q}}$, gluinos $\tilde{\mathrm{g}}$, neutralinos and charginos $\tilde{\chi}$, and Higgs states $\mathrm{h}^{0}, \mathrm{H}^{0}, \mathrm{~A}^{0}$ and $\mathrm{H}^{ \pm}$. The idea, however, is that these situations could represent also a number of other non-standard particles. For instance, the decay of a spin 0 leptoquark $\mathrm{L}_{\mathrm{Q}} \rightarrow \mathrm{q} \ell$ is closely similar to $\tilde{\mathrm{q}} \rightarrow \mathrm{q} \tilde{\chi}$.

All calculations have been performed in the zero-width limit of the decaying state and the decay products, in order to allow a gauge invariant separation of radiation in the production and decay stages. (As explained above, the Monte Carlo simulation of processes does include mass selection according to the appropriate Breit-Wigners, so we 


\begin{tabular}{|c|c|c|c|c|}
\hline colour & spin & $\gamma_{5}$ & example & codes \\
\hline $1 \rightarrow 3+\overline{3}$ & - & - & $($ eikonal) & $6-9$ \\
$1 \rightarrow 3+\overline{3}$ & $1 \rightarrow \frac{1}{2}+\frac{1}{2}$ & $1, \gamma_{5}, 1 \pm \gamma_{5}$ & $\mathrm{Z}^{0} \rightarrow \mathrm{q} \overline{\mathrm{q}}$ & $11-14$ \\
$3 \rightarrow 3+1$ & $\frac{1}{2} \rightarrow \frac{1}{2}+1$ & $1, \gamma_{5}, 1 \pm \gamma_{5}$ & $\mathrm{t} \rightarrow \mathrm{bW}^{+}$ & $16-19$ \\
$1 \rightarrow 3+\overline{3}$ & $0 \rightarrow \frac{1}{2}+\frac{1}{2}$ & $1, \gamma_{5}, 1 \pm \gamma_{5}$ & $\mathrm{H}^{0} \rightarrow \mathrm{q} \overline{\mathrm{q}}$ & $21-24$ \\
$3 \rightarrow 3+1$ & $\frac{1}{2} \rightarrow \frac{1}{2}+0$ & $1, \gamma_{5}, 1 \pm \gamma_{5}$ & $\mathrm{t} \rightarrow \mathrm{bH}^{+}$ & $26-29$ \\
$1 \rightarrow 3+\overline{3}$ & $1 \rightarrow 0+0$ & 1 & $\mathrm{Z}^{0} \rightarrow \tilde{\mathrm{q}} \overline{\tilde{\mathrm{q}}}$ & $31-34$ \\
$3 \rightarrow 3+1$ & $0 \rightarrow 0+1$ & 1 & $\tilde{\mathrm{q}} \rightarrow \tilde{\mathrm{q}}^{\prime} \mathrm{W}^{+}$ & $36-39$ \\
$1 \rightarrow 3+\overline{3}$ & $0 \rightarrow 0+0$ & 1 & $\mathrm{H}^{0} \rightarrow \tilde{\mathrm{q}} \overline{\tilde{q}}$ & $41-44$ \\
$3 \rightarrow 3+1$ & $0 \rightarrow 0+0$ & 1 & $\tilde{\mathrm{q}} \rightarrow \tilde{\mathrm{q}}^{\prime} \mathrm{H}^{+}$ & $46-49$ \\
$1 \rightarrow 3+\overline{3}$ & $\frac{1}{2} \rightarrow \frac{1}{2}+0$ & $1, \gamma_{5}, 1 \pm \gamma_{5}$ & $\tilde{\chi} \rightarrow \mathrm{q} \tilde{\tilde{q}}$ & $51-54$ \\
$3 \rightarrow 3+1$ & $0 \rightarrow \frac{1}{2}+\frac{1}{2}$ & $1, \gamma_{5}, 1 \pm \gamma_{5}$ & $\tilde{\mathrm{q}} \rightarrow \mathrm{q} \tilde{\chi}$ & $56-59$ \\
$3 \rightarrow 3+1$ & $\frac{1}{2} \rightarrow 0+\frac{1}{2}$ & $1, \gamma_{5}, 1 \pm \gamma_{5}$ & $\mathrm{t} \rightarrow \tilde{\mathrm{t}} \tilde{\chi}$ & $61-64$ \\
$8 \rightarrow 3+\overline{3}$ & $\frac{1}{2} \rightarrow \frac{1}{2}+0$ & $1, \gamma_{5}, 1 \pm \gamma_{5}$ & $\tilde{\mathrm{g}} \rightarrow \mathrm{q} \tilde{\tilde{\mathrm{q}}}$ & $66-69$ \\
$3 \rightarrow 3+8$ & $0 \rightarrow \frac{1}{2}+\frac{1}{2}$ & $1, \gamma_{5}, 1 \pm \gamma_{5}$ & $\tilde{\mathrm{q}} \rightarrow \mathrm{q} \tilde{\mathrm{g}}$ & $71-74$ \\
$3 \rightarrow 3+8$ & $\frac{1}{2} \rightarrow 0+\frac{1}{2}$ & $1, \gamma_{5}, 1 \pm \gamma_{5}$ & $\mathrm{t} \rightarrow \tilde{\mathrm{t}} \tilde{\mathrm{g}}$ & $76-79$ \\
$1 \rightarrow 8+8$ & $\rightarrow-$ & $(\mathrm{eikonal})$ & $81-84$ \\
\hline
\end{tabular}

Table 1: The processes that have been calculated, also with one extra gluon in the final state. Colour is given with 1 for singlet, 3 for triplet and 8 for octet. See the text for an explanation of the $\gamma_{5}$ column and further comments.

here only comment on the width dependence of the additional gluon radiation, i.e. in the ratio of first to leading order cross sections.) Such a separation occurs naturally for exchanged colourless particles, so is then no problem. For a coloured particle of width $\Gamma$, this is a poor approximation in the region of gluon energies (in the particle rest frame) below $\Gamma$. The $t$ is still sufficiently narrow that the production-decay interference is not a major problem, see above, but for heavier squarks and gluinos a more complex description may be required. The lowest coloured SUSY states, e.g. stop, tend to have small widths, however, and it is on such states we will concentrate our studies. The additional complications for very wide particles will be deferred to some future study.

The classification by colour and spin is fairly obvious, but that does not completely specify the structure of the process. Consider e.g. $\mathrm{e}^{+} \mathrm{e}^{-} \rightarrow \gamma^{*} / \mathrm{Z}^{0} \rightarrow \mathrm{q} \overline{\mathrm{q}}$. The cross section (neglecting mass effects) is then

$$
\sigma_{0} \propto e_{\mathrm{e}}^{2} e_{\mathrm{q}}^{2}+2 e_{\mathrm{e}} v_{\mathrm{e}} \Re \mathrm{e} \Xi e_{\mathrm{q}} v_{\mathrm{q}}+\left(v_{\mathrm{e}}^{2}+a_{\mathrm{e}}^{2}\right)|\Xi|^{2}\left(v_{\mathrm{q}}^{2}+a_{\mathrm{q}}^{2}\right)
$$

where

$$
\Xi=\frac{1}{16 \sin ^{2} \theta_{W} \cos ^{2} \theta_{W}} \frac{s}{s-m_{\mathrm{Z}}^{2}+i m_{\mathrm{Z}} \Gamma_{\mathrm{Z}}}
$$

represents the ratio between the $\mathrm{Z}^{0}$ and the $\gamma$ propagators and couplings. The term proportional to $a_{\mathrm{q}}^{2}$ corresponds to the $\mathrm{q} \overline{\mathrm{q}}$ pair coming from an axial vector source, the 
rest to it coming from a vector source. Since the QCD radiation from these two is somewhat different, we need to include the proper mixture, which depends on the CM energy, $s=E_{\mathrm{CM}}^{2}$. It has then been simplest to perform the calculations for a pure vector source and a pure axial vector source, represented by 1 and $\gamma_{5}$ in Table $\mathbb{1}$, and mix in the proportions required. We note that this mixing strategy is possible since the total cross section does not contain any interference terms of the character $v_{\mathrm{q}} a_{\mathrm{q}}$. (Such terms do arise when the forward-backward charge asymmetry is considered.) The pure left-handed mixture $V-A=1-\gamma_{5}$ is of special interest, since it represents the $\mathrm{W}^{ \pm}$bosons. Therefore it has been calculated separately, and is denoted by $1 \pm \gamma_{5}$ in Table 1; as we already noted the sign (of the interference term in the squared matrix element) is irrelevant for the QCD emission aspects. In total, four alternatives are therefore open in our implementation: to have a pure vector source, a pure axial vector, an arbitrary mixture $\alpha V+(1-\alpha) A$ and the special equal mixture. In this order, that gives the four codes $11-14$ in Table 1, according to the numbering scheme used in the PYTHIA function PYMAEL introduced in version 6.153. (This routine takes as input the process code, $x_{1}, x_{2}, r_{1}, r_{2}$ and $\alpha$, and returns the ratio $\left(1 / \sigma_{0}\right) \mathrm{d} \sigma / \mathrm{d} x_{1} \mathrm{~d} x_{2}$, omitting a factor of $\left(\alpha_{\mathrm{s}} / 2 \pi\right) C_{F}$.)

Correspondingly, also most other processes can come either with or without $\gamma_{5}$ factors in the amplitude, or in arbitrary mixtures thereof. In the Higgs sector, normally the $\mathrm{h}^{0}$ and $\mathrm{H}^{0}$ are scalar, the $\mathrm{A}^{0}$ pseudoscalar and the $\mathrm{H}^{ \pm}$a parameter-dependent mixture of 1 and $\gamma_{5}$. If the coupling structure is generalized, also the neutral Higgses could be mixtures, however. The $\tilde{\mathrm{q}}_{L}$ and $\tilde{\mathrm{q}}_{R}$ squark partners of the left- and right-handed quarks come with wave function factors $1-\gamma_{5}$ and $1+\gamma_{5}$, respectively. The squark mass eigenstates will be mixtures of these, with significant mixing expected especially in the third generation. With two squarks in a process, $\gamma_{5}^{2}=1$ ensures that the matrix element still can be written as a sum $\alpha 1+(1-\alpha) \gamma_{5}$. Again, therefore, we have chosen to perform most of the calculations with and without a $\gamma_{5}$ factor and then leave open to have the mixing depend on the current parameter choice. As a further simplification, the $1 \pm \gamma_{5}$ mixture is used whenever the correct choice is not known, since this mixture represents an average behaviour. In some instances, further restrictions exist, e.g. a pseudoscalar cannot decay to two scalars, at least among the MSSM processes at our disposal.

The process at the top of Table 11 is the spin-independent eikonal answer of eq. (18), extended from the soft region where it is intended to be valid:

$$
\begin{aligned}
\frac{1}{\sigma_{0}} \frac{\mathrm{d} \sigma}{\mathrm{d} x_{1} \mathrm{~d} x_{2}}= & \frac{\alpha_{\mathrm{s}}}{2 \pi} C_{F}\left\{\frac{2\left(x_{1}+x_{2}-1-r_{1}^{2}-r_{2}^{2}\right)}{\left(1+r_{1}^{2}-r_{2}^{2}-x_{1}\right)\left(1+r_{2}^{2}-r_{1}^{2}-x_{2}\right)}\right. \\
& \left.-\frac{2 r_{2}^{2}}{\left(1+r_{2}^{2}-r_{1}^{2}-x_{2}\right)^{2}}-\frac{2}{\left(1+r_{1}^{2}-r_{2}^{2}-x_{1}\right)^{2}}\right\} .
\end{aligned}
$$

The first numerator, $2\left(x_{1}+x_{2}-1-r_{1}^{2}-r_{2}^{2}\right)$, here is based on an evaluation of the $2 p_{1} p_{2}$ numerator of eq. (18) with $p_{1}$ and $p_{2}$ given by their values after the emission of $p_{3}$. Away from the soft-gluon limit, however, there is some leeway in this assumption. If instead the $p_{1}$ and $p_{2}$ values before the emission of $p_{3}$ had been used, one would have obtained $x_{1}+x_{2}-1=1-x_{3} \rightarrow 1$. Such a seemingly minor substitution has quite dramatic effects for collinear emission even at rather small $x_{3}$, however, and is not really an option. In order to have a not too unrealistic alternative to compare with, we therefore only allow deviations proportional to $x_{3}^{2}$. (This is also what comes out of the $x_{1}^{2}+x_{2}^{2}$ numerator of the massless process $V \rightarrow \mathrm{q} \overline{\mathrm{q} g}$.) The extreme in this direction would be $x_{1}+x_{2}-1 \rightarrow 1-x_{3}+x_{3}^{2}$, but we will also allow arbitrary admixtures $x_{1}+x_{2}-1 \rightarrow 1-x_{3}+\alpha x_{3}^{2}$, with $\alpha$ as a free parameter, obviously in no physics relation to the $\alpha$ introduced above. 
In all the formulae, the decay product mass ratios are kept as free parameters, $r_{1}=$ $m_{b} / m_{a}$ and $r_{2}=m_{c} / m_{a}$, while the gluon is massless. Since we are not interested in cross sections per se, but in the probability for gluon emission, in the LO cross sections only the mass dependence is retained, normalized to unity for $r_{1}=r_{2}=0$, e.g.

$$
\sigma_{0}\left(V, A \rightarrow \mathrm{q}_{1} \overline{\mathrm{q}}_{2}\right)=\frac{1}{2}\left\{2-\left(r_{1}^{2}-r_{2}^{2}\right)^{2}-r_{1}^{2}-r_{2}^{2} \pm 6 r_{1} r_{2}\right\} \sqrt{\left(1-r_{1}^{2}-r_{2}^{2}\right)^{2}-4 r_{1}^{2} r_{2}^{2}} .
$$

What is omitted is then some set of couplings and propagators. Exactly the same set is also omitted from the first-order $\mathrm{d} \sigma / \mathrm{d} x_{1} \mathrm{~d} x_{2}$, thereby leaving the ratio unchanged. Additionally the common factor $\left(\alpha_{\mathrm{s}} / 2 \pi\right) C_{F}$, is omitted, to leave the choice of $\alpha_{\mathrm{s}}\left(p_{\perp}^{2}\right)$ free to be made elsewhere. The ratio of the first to leading order cross sections then gives the assumed differential gluon-emission rate. In the case of a mixture without and with $\gamma_{5}$ factors, the sum has to be taken for numerator and denominator separately, e.g.

$$
\frac{1}{\sigma_{0}} \frac{\mathrm{d} \sigma}{\mathrm{d} x_{1} \mathrm{~d} x_{2}}\left(\mathrm{e}^{+} e^{-} \rightarrow \gamma^{*} / \mathrm{Z}^{0} \rightarrow \mathrm{q} \overline{\mathrm{q}}\right)=\frac{\alpha \mathrm{d} \sigma^{V} / \mathrm{d} x_{1} \mathrm{~d} x_{2}+(1-\alpha) \mathrm{d} \sigma^{A} / \mathrm{d} x_{1} \mathrm{~d} x_{2}}{\alpha \sigma_{0}^{V}+(1-\alpha) \sigma_{0}^{A}} .
$$

The vector fraction $\alpha$ can here be read off from the lowest-order expression in eq. (42), where the mass factors of eq. (45) were omitted. The mass effects are instead included in the individual $\mathrm{d} \sigma / \mathrm{d} x_{1} \mathrm{~d} x_{2}$ and $\sigma_{0}$ terms of eq. (46). This standard e.g. means that $V-A$ corresponds to $\alpha=1 / 2$, which then because of mass effects gives a somewhat larger vector than axial fraction.

Note that first-order corrections to the total cross section are not included in the $\sigma_{0}$ denominator. This is not a unique choice, but a rather natural one: if we consider the gluon emission rate as the ratio of two cross sections, then including $\mathcal{O}\left(\alpha_{\mathrm{s}}\right)$ corrections to one but not the other is not likely to improve the overall accuracy of the calculation. In the soft-gluon limit, it would even break the spin independence of the radiation pattern (cf. the next subsection), i.e. give the wrong physics. And since a complete one-loop calculation of both quantities is well beyond the scope of this article, we remain with lowest non-trivial order for both quantities. Furthermore, if the total cross section is written in the form $\sigma_{\text {tot }}=\sigma_{0}\left(1+a \alpha_{\mathrm{s}} / \pi\right)$, then decay rates calculated so far tend to give $a$ values of order unity [28, 29], i.e. small effects. An exception would be Coulomb corrections in the threshold region, but there the phase space for real gluon emission is vanishingly small anyway, so of no physical interest. By contrast, one-loop corrections to three-jet rates tend to be larger [30], although most of that is absorbed by the partonshower choice of a smaller kinematics-dependent scale like $p_{\perp}^{2}$ [30, 31].

\subsection{Radiation patterns}

Before applying the matrix elements to specific physics situations, it is interesting to compare them between each other under similar conditions. This will provide an understanding of the amount of spin and colour dependence in the matrix elements, and thus the extent to which a process-dependent Monte Carlo implementation can be expected to provide an improvement over a process-blind one, e.g. based on some simple dead cone formula.

Fig. 3 is intended to provide a first qualitative glimpse of differences. The amount of detail may be bewildering, but at this point the idea is to bring up differences and similarities in a broad sense, without concentrating on each process specifically.

First consider the full curves in Figs. 3a-c, which are all for the colour structure $1 \rightarrow 3+\overline{3}$ and the same kinematics, and only differ by the spin pattern: $1 \rightarrow 1 / 2+1 / 2$, 

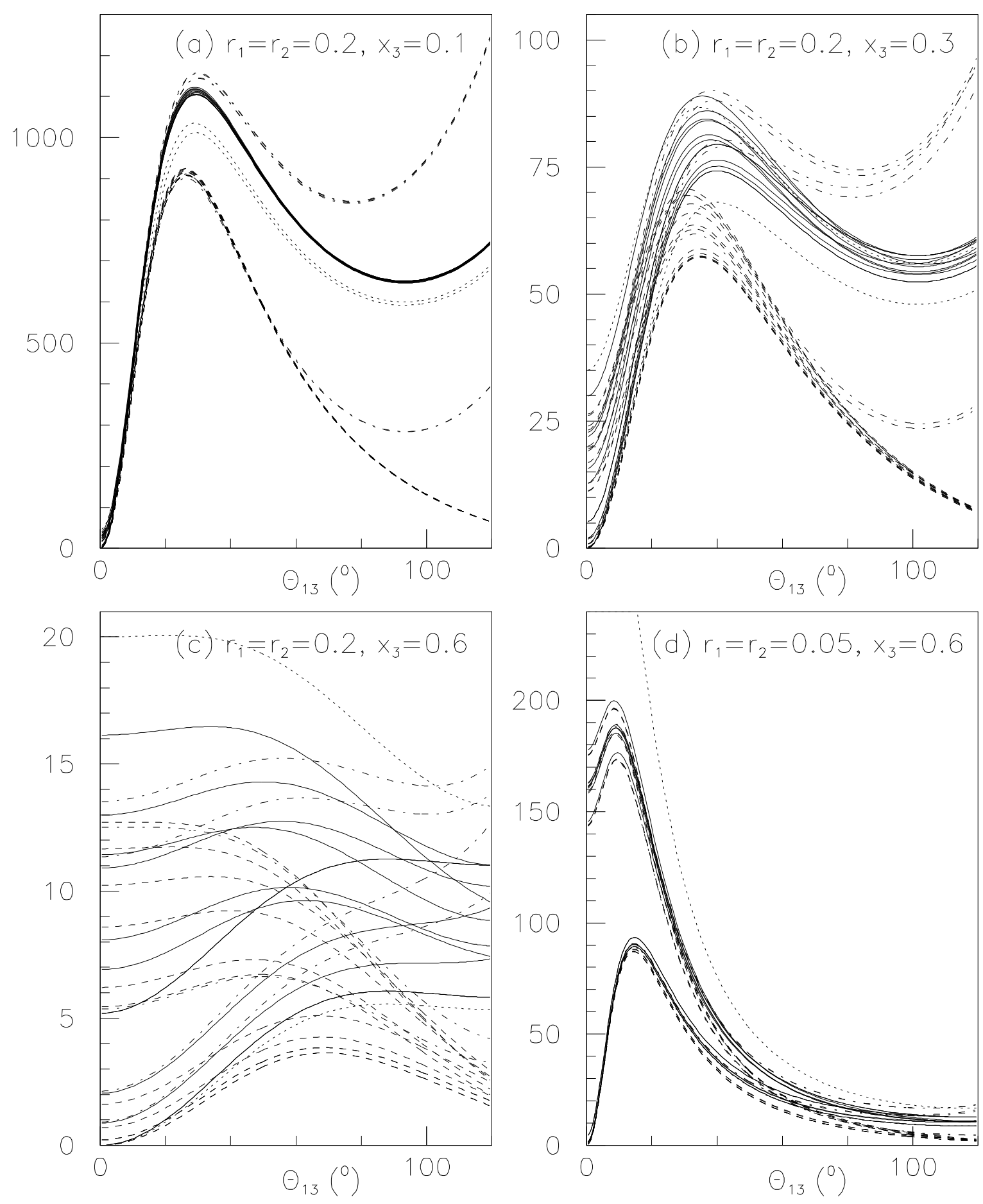

Figure 3: The gluon emission rate as a function of the emission angle $\theta_{13}$. Specifically, the vertical axis gives $\left(1 / \sigma_{0}\right) \mathrm{d} \sigma / \mathrm{d} x_{1} \mathrm{~d} x_{2}$ with a normalization factor $\left(\alpha_{\mathrm{s}} / 2 \pi\right) C_{F}$ removed. This three-jet phase space density differs from $\mathrm{d} \sigma / \mathrm{d} \theta_{13} \mathrm{~d} x_{3}$ by a simple Jacobian. A variety of different processes are compared: decay of a colour singlet to a triplet plus an antitriplet full curves, ditto in the eikonal approximation dotted, decay of a triplet to a triplet plus singlet dashed, and gluino processes dash-dotted. The four frames differ in the scaled masses $r_{i}=m_{i} / E_{\mathrm{CM}}$ of the two decay products, and in the gluon energy fraction $x_{3}$. Further explanations are given in the text. 
$0 \rightarrow 1 / 2+1 / 2,1 \rightarrow 0+0,0 \rightarrow 0+0$, and $1 / 2 \rightarrow 1 / 2+0$, in relevant cases with and without a $\gamma_{5}$ (but no intermediate mixtures such as $1 \pm \gamma_{5}$ ). In the first frame, where the gluon is soft, these curves almost completely overlap. At smaller $x_{3}$ the agreement becomes even better, and one can then truly speak of a universal soft-gluon emission pattern, with a characteristic dead cone of opening angle $\theta \approx 2 r=0.4=23^{\circ}$ in this example. By contrast, when $x_{3}$ is increased, the curves tend to disagree more and more. At $x_{3}=0.3$, the dead cone is still visible, although it is starting to fill in, but at $x_{3}=0.6$ it is completely gone. Note that the vertical scale changes significantly between the three frames; in absolute numbers the differences between the curves is about a factor two larger at $x_{3}=0.1$ than at 0.6 , but in relative terms the differences are negligible at small $x_{3}$.

This should bring home the message that the dead cone concept can only be used at small gluon energies, and is irrelevant for energetic gluons. It is only the lowest of the full curves, the completely spinless process $0 \rightarrow 0+0$, that does preserve the exact dead cone concept, i.e. has a cross section that always vanishes in the collinear limit (in the rest frame of the decaying particle).

The lower dotted curve is the eikonal expression of eq. (44), which is constructed to have an exact dead cone. It does agree fairly well with the spinless process, but not with anything else. The upper dotted curve is the modified eikonal, with an $x_{3}^{2}$ term added. It tends to overshoot all processes at small angles, while some intermediate mixture, $\alpha \approx 0.5$, would do a sensible job for many processes in the small-angle region. For medium small gluon energies and large angles, both eikonal forms undershoot, however. In general, one may conclude that the eikonal formula is not particularly useful for practical considerations, and that the process-specific matrix elements need to be used.

Of course, the detailed pattern depends on the masses. In Fig. 3 $3 \mathrm{~d}$ these are lower than in the first three, and representative for $\mathrm{Z}^{0} \rightarrow \mathrm{b} \overline{\mathrm{b}}$. Then the dead cone concept works up to somewhat larger gluon energies, and a trace of it is still left at $x_{3}=0.6$. It is again preserved exactly for $0 \rightarrow 0+0$ and the eikonal, and also approximately for $1 \rightarrow 0+0$.

In order to quantify the difference in the total amount of gluon radiation, we compare three measures on the three-jet phase space. Since the total three-jet cross section contains a soft-gluon divergence, this soft region has to be avoided. One measure is thus to integrate the total amount of radiated gluon energy, in shorthand

$$
\int x_{3}=\int x_{3} \frac{1}{\sigma_{0}} \frac{\mathrm{d} \sigma}{\mathrm{d} x_{1} \mathrm{~d} x_{2}} \mathrm{~d} x_{1} \mathrm{~d} x_{2} .
$$

Correspondingly we define a shorthand $\int\left(1-x_{1}\right)\left(1-x_{2}\right)$ as an alternative removal of the denominator of the matrix elements (generalized to $\int\left(1+r_{1}^{2}-r_{2}^{2}-x_{1}\right)\left(1+r_{2}^{2}-r_{1}^{2}-x_{2}\right)$ for unequal masses). Finally a Durham distance [32] $y_{i j}=\min \left(x_{i}^{2}, x_{j}^{2}\right)\left(1-\cos \theta_{i j}\right) / 2$ is used to define a hard three-jet region for which $y_{\mathrm{D}}=\min \left(y_{12}, y_{13}, y_{23}\right)>0.1$ and a corresponding three-jet rate $\int \theta\left(y_{\mathrm{D}}-0.1\right)$, where $\theta$ is the step function. To simplify a comparison between the processes, all results have arbitrarily been normalized to those for the $V \rightarrow \mathrm{q} \overline{\mathrm{q}}$ process.

As can be seen in Table 2, the three measures give about the same message, namely that differences between the processes are significant. In $\int x_{3}$ the ratio between the two extremes is a factor 1.30, in $\int\left(1-x_{1}\right)\left(1-x_{2}\right) 1.70$, and in $\int \theta\left(y_{\mathrm{D}}-0.1\right) 1.91$. This shows a steady progression of larger ratios the more one is biased towards the hard three-jet region. The eikonal expression is below all the calculated processes, and the modified eikonal (full blast) above.

The results in Table 2 are for the case where the daughter masses constitute a significant fraction of the energy available. The other limit, where instead the daughters are 


\begin{tabular}{|c|c|c|c|c|c|}
\hline colour & spin & $\gamma_{5}$ & $\int x_{3}$ & $\int\left(1-x_{1}\right)\left(1-x_{2}\right)$ & $\int \theta\left(y_{\mathrm{D}}-0.1\right)$ \\
\hline \multirow[t]{8}{*}{$1 \rightarrow 3+\overline{3}$} & \multirow{2}{*}{$1 \rightarrow \frac{1}{2}+\frac{1}{2}$} & 1 & 1.000 & 1.000 & 1.000 \\
\hline & & $\gamma_{5}$ & 1.056 & 1.112 & 1.133 \\
\hline & \multirow{2}{*}{$0 \rightarrow \frac{1}{2}+\frac{1}{2}$} & 1 & 1.134 & 1.293 & 1.376 \\
\hline & & $\gamma_{5}$ & 1.093 & 1.207 & 1.271 \\
\hline & $1 \rightarrow 0+0$ & 1 & 1.073 & 1.205 & 1.310 \\
\hline & $0 \rightarrow 0+0$ & 1 & 0.875 & 0.758 & 0.720 \\
\hline & \multirow[t]{2}{*}{$\frac{1}{2} \rightarrow \frac{1}{2}+0$} & 1 & 0.953 & 0.918 & 0.916 \\
\hline & & $\gamma_{5}$ & 1.057 & 1.132 & 1.179 \\
\hline \multirow[t]{2}{*}{$1 \rightarrow 3+\overline{3}$} & \multirow{2}{*}{$\begin{array}{c}\text { eikonal } \\
\text { eikonal }+x_{3}^{2}\end{array}$} & - & 0.802 & 0.695 & 0.659 \\
\hline & & - & 1.201 & 1.518 & 1.670 \\
\hline \multirow[t]{10}{*}{$3 \rightarrow 3+1$} & \multirow{2}{*}{$\frac{1}{2} \rightarrow \frac{1}{2}+1$} & 1 & 0.323 & 0.306 & 0.287 \\
\hline & & $\gamma_{5}$ & 0.356 & 0.365 & 0.349 \\
\hline & \multirow{2}{*}{$\frac{1}{2} \rightarrow \frac{1}{2}+0$} & 1 & 0.312 & 0.284 & 0.258 \\
\hline & & $\gamma_{5}$ & 0.357 & 0.363 & 0.344 \\
\hline & $0 \rightarrow 0+1$ & 1 & 0.287 & 0.242 & 0.218 \\
\hline & $0 \rightarrow 0+0$ & 1 & 0.279 & 0.224 & 0.194 \\
\hline & \multirow[t]{2}{*}{$0 \rightarrow \frac{1}{2}+\frac{1}{2}$} & 1 & 0.359 & 0.379 & 0.375 \\
\hline & & $\gamma_{5}$ & 0.347 & 0.354 & 0.346 \\
\hline & \multirow[t]{2}{*}{$\frac{1}{2} \rightarrow 0+\frac{1}{2}$} & 1 & 0.294 & 0.257 & 0.239 \\
\hline & & $\gamma_{5}$ & 0.314 & 0.302 & 0.298 \\
\hline \multirow[t]{4}{*}{$3 \rightarrow 3+8$} & \multirow[t]{2}{*}{$0 \rightarrow \frac{1}{2}+\frac{1}{2}$} & 1 & 1.634 & 1.833 & 1.922 \\
\hline & & $\gamma_{5}$ & 1.574 & 1.712 & 1.775 \\
\hline & \multirow[t]{2}{*}{$\frac{1}{2} \rightarrow 0+\frac{1}{2}$} & 1 & 1.385 & 1.320 & 1.291 \\
\hline & & $\gamma_{5}$ & 1.549 & 1.664 & 1.675 \\
\hline \multirow[t]{2}{*}{$8 \rightarrow 3+\overline{3}$} & \multirow[t]{2}{*}{$\frac{1}{2} \rightarrow \frac{1}{2}+0$} & 1 & 0.561 & 0.493 & 0.445 \\
\hline & & $\gamma_{5}$ & 0.621 & 0.607 & 0.574 \\
\hline
\end{tabular}

Table 2: Three measures on the amount of gluon radiation in different processes, for mass ratios $r_{1}=r_{2}=0.2$. For clarity, results have been normalized to the process in the top line. See the text for a detailed explanation.

massless, is shown in Table 3. The main message is that the process dependence remains also in the latter case, even if normally reduced in magnitude by about a factor of two between the extremes. The detailed picture is not so simple, however. Some processes agree in the massless limit when they do not for nonvanishing masses while, in the other extreme, others disagree even more for vanishing masses. 


\begin{tabular}{|c|c|c|c|}
\hline \multicolumn{1}{|c|}{ colour } & spin & $\int\left(1-x_{1}\right)\left(1-x_{2}\right)$ & $\int \theta\left(y_{\mathrm{D}}-0.1\right)$ \\
\hline $1 \rightarrow 3+\overline{3}$ & $1 \rightarrow \frac{1}{2}+\frac{1}{2}$ & 1.000 & 1.000 \\
& $0 \rightarrow \frac{1}{2}+\frac{1}{2}$ & 1.167 & 1.184 \\
& $1 \rightarrow 0+0$ & 1.000 & 1.141 \\
& $0 \rightarrow 0+0$ & 0.667 & 0.773 \\
& $\frac{1}{2} \rightarrow \frac{1}{2}+0$ & 0.917 & 0.979 \\
\hline $1 \rightarrow 3+\overline{3}$ & eikonal & 0.667 & 0.773 \\
& eikonal $+x_{3}^{2}$ & 1.667 & 1.595 \\
\hline $3 \rightarrow 3+1$ & $\frac{1}{2} \rightarrow \frac{1}{2}+1$ & 0.347 & 0.282 \\
& $\frac{1}{2} \rightarrow \frac{1}{2}+0$ & 0.347 & 0.282 \\
& $0 \rightarrow 0+1$ & 0.222 & 0.214 \\
& $0 \rightarrow 0+0$ & 0.222 & 0.214 \\
& $0 \rightarrow \frac{1}{2}+\frac{1}{2}$ & 0.389 & 0.328 \\
& $\frac{1}{2} \rightarrow 0+\frac{1}{2}$ & 0.264 & 0.260 \\
\hline $3 \rightarrow 3+\overline{3}$ & $\frac{1}{2} \rightarrow \frac{1}{2}+0$ & 0.573 & 1.659 \\
& $0 \rightarrow \frac{1}{2}+\frac{1}{2}$ & 1.701 & 1.384 \\
\hline $3+8$ & $\frac{1}{2} \rightarrow 0+\frac{1}{2}$ & 1.389 & 0.487 \\
\hline
\end{tabular}

Table 3: Two measures on the amount of gluon radiation in different processes, for mass ratios $r_{1}=r_{2}=0$, cf. Table 2. For massless daughters the $\gamma_{5}$ factor makes no difference, and so those results are not shown separately. The $\int x_{3}$ measure now is collinear divergent and therefore not shown.

The set of processes with colour structure $3 \rightarrow 3+1$ can be viewed as crossed versions of the $1 \rightarrow 3+\overline{3}$ ones, but the differences in kinematics result in another overall picture. Examples of radiation patterns are shown by dashed curves in Fig. 3. The small-angle behaviour displays the universal dead cone effect at small $x_{3}$ and again diverges wildly at larger $x_{3}$. Note that we have oriented all processes so that the radiating daughter colour charge is at $0^{\circ}$, wherefore the radiation continues to drop off at large angles. This is unlike the previous set of curves, which turn around at or near the mid angle to the other radiating daughter. (For small $x_{3}$ the two daughters are almost back-to-back, i.e. a bisector at $90^{\circ}$, while an $x_{3}$ of 0.6 allows an almost symmetric configuration with $120^{\circ}$ between all three.)

It is here interesting to remind of the QED answer for the process $V \rightarrow \mathrm{f} \bar{f} \gamma$ with $m_{\mathrm{f}}=m_{\overline{\mathrm{f}}}=0$

$$
\frac{1}{\sigma_{0}} \frac{\mathrm{d} \sigma}{\mathrm{d} x_{1} \mathrm{~d} x_{2}}=\frac{\alpha_{\mathrm{em}}}{2 \pi} \frac{x_{1}^{2}+x_{2}^{2}}{\left(1-x_{1}\right)\left(1-x_{2}\right)}\left(e_{\mathrm{f}} \frac{1-x_{1}}{x_{3}}-e_{\overline{\mathrm{f}}} \frac{1-x_{2}}{x_{3}}\right)^{2},
$$

where $e_{V}=e_{\mathrm{f}}+e_{\overline{\mathrm{f}}}$ has been used to eliminate the explicit appearance of terms corresponding to radiation off the $V$. For $e_{V}=0, e_{\mathrm{f}}=-e_{\overline{\mathrm{f}}}$ this is the QED analogue of eq. (9), 


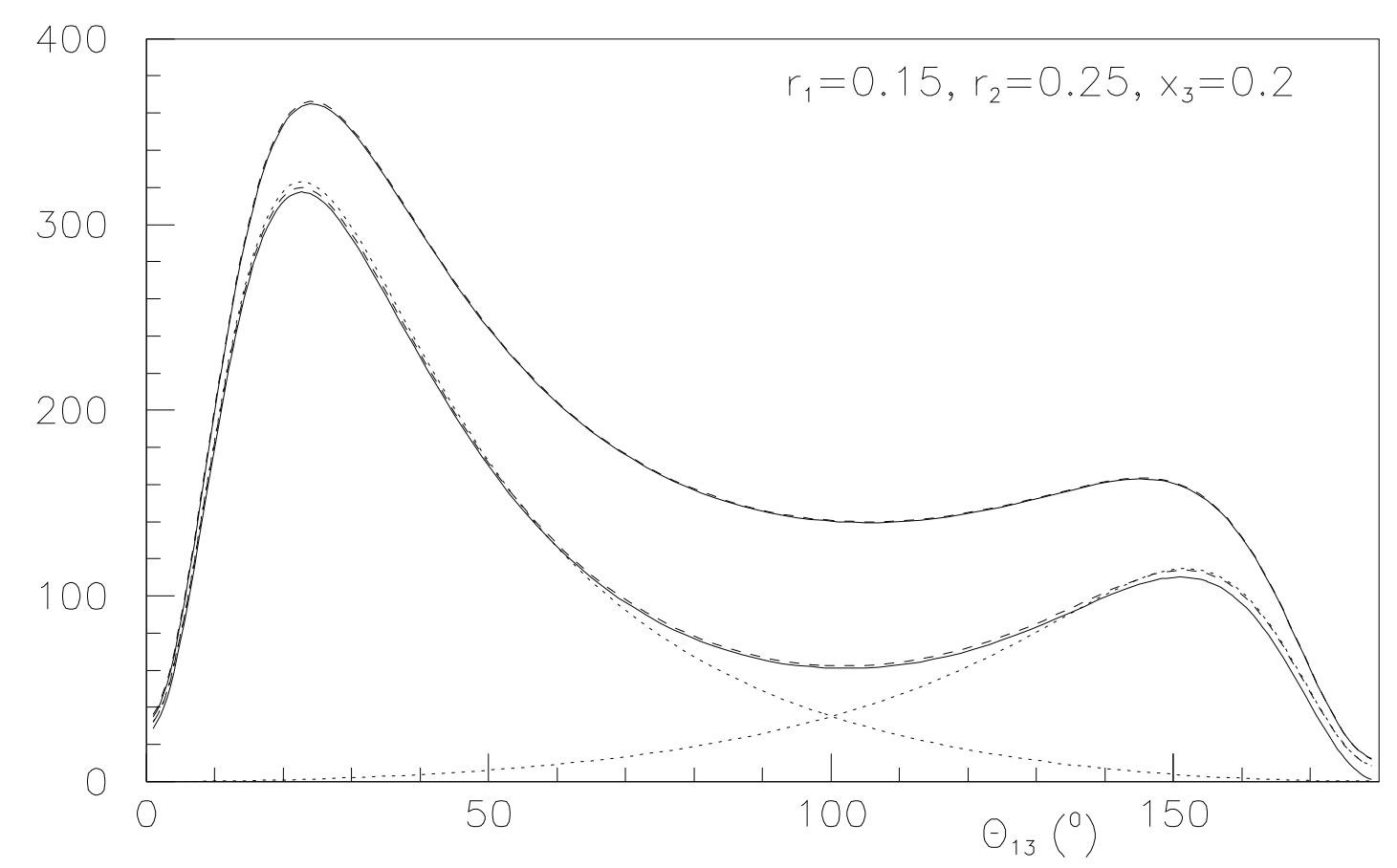

Figure 4: Test of the additivity of gluon emission rates, $\mathrm{d} \sigma / \mathrm{d} x_{1} \mathrm{~d} x_{2}$ with a normalization factor $\sigma_{0} C_{F} \alpha_{\mathrm{s}} / 2 \pi$ removed. The dotted curves show two processes with colour flow $3 \rightarrow 3+1$ and $3 \rightarrow 1+3$, respectively (and spin $1 / 2 \rightarrow 1 / 2+1$ ). The upper and lower full curves give the complete expressions for $1 \rightarrow 3+\overline{3}$ ( $\operatorname{spin} 1 \rightarrow 1 / 2+1 / 2$ ) and $8 \rightarrow 3+\overline{3}$ ( spin $1 / 2 \rightarrow 1 / 2+0$ ) processes, respectively. The two dashed curves, almost completely hidden by the full ones, are the same processes according to the additive approximations in eq. (50).

while the current case corresponds to $e_{\overline{\mathrm{f}}}=0, \alpha_{\mathrm{em}} e_{\mathrm{f}}^{2} \rightarrow \alpha_{\mathrm{s}} C_{F}$ :

$$
\frac{1}{\sigma_{0}} \frac{\mathrm{d} \sigma}{\mathrm{d} x_{1} \mathrm{~d} x_{2}}=\frac{\alpha_{\mathrm{s}}}{2 \pi} C_{F} \frac{x_{1}^{2}+x_{2}^{2}}{\left(1-x_{1}\right)\left(1-x_{2}\right)}\left(\frac{1-x_{1}}{x_{3}}\right)^{2}=\frac{\alpha_{\mathrm{s}}}{2 \pi} C_{F} \frac{x_{1}^{2}+x_{2}^{2}}{x_{3}\left(1-x_{2}\right)} \frac{1-x_{1}}{x_{3}} .
$$

Here the first part of the final expression essentially is the shower answer of eq. (36). In the soft-gluon limit $\left(1-x_{1}\right) / x_{3} \approx\left(1+\cos \theta_{13}\right) / 2 \approx\left(1-\cos \theta_{23}\right) / 2$, so this extra factor gives a further dampening at large emission angles, above the shower ansatz (which in itself is dampened by one such angular factor relative to the colour singlet decay). A main consequence of this extra factor is that the three-jet activity in a colour $3 \rightarrow 3+1$ event is less than half of that of a $1 \rightarrow 3+\overline{3}$ one, a pattern which remains when masses are included, see Table 2 .

Finally we come to the processes with a gluino, denoted by dash-dotted lines in Fig. 3 . In the limit of infinitely many colours $N_{C}$, the process $3 \rightarrow 3+8$ may be viewed as one colour flowing through from the initial triplet to the gluino, and a different colouranticolour pair created between the final triplet and the gluino, i.e. as the sum of $3 \rightarrow 1+3$ and $1 \rightarrow 3+\overline{3}$. Interference terms between the two colour flows would be suppressed by a factor $1 / N_{C}^{2}$, just as in the radiation pattern for the colour-related process $V \rightarrow \mathrm{q} \overline{\mathrm{q} g}$ 《19. This process therefore shows the most three-jet activity of the ones studied, especially in the not-displayed gluino hemisphere.

By the same token, $8 \rightarrow 3+\overline{3}$ may be approximated by the incoherent sum of a $3 \rightarrow 3+1$ and a $\overline{3} \rightarrow 1+\overline{3}$ radiation pattern, which gives less radiation than the $1 \rightarrow 3+\overline{3}$ 
processes. The QED formula, eq. (48), may here offer a convenient starting point. The $1 \rightarrow 3+\overline{3}$ process corresponds to $e_{\mathrm{f}}=-e_{\overline{\mathrm{f}}}$, so that the interference term $-e_{\mathrm{f}} e_{\overline{\mathrm{f}}}$ is positive. The $8 \rightarrow 3+\overline{3}$ process, e.g. $\tilde{\mathrm{g}} \rightarrow \mathrm{q} \overline{\tilde{\mathrm{q}}}$, can instead be emulated in QED by $e_{\mathrm{f}}=e_{\overline{\mathrm{f}}}$, i.e. a doubly-charged gluino [34]. The interference term then is negative, although suppressed by a colour factor $1 / N_{C}^{2}$ in the QCD case.

Thus, given a radiation pattern $f_{1}\left(\theta_{13}\right)$ for emission off parton 1 like in a colour $3 \rightarrow$ $3+1$ process, and a corresponding $f_{2}\left(\theta_{13}\right)$ for a colour $3 \rightarrow 1+3$ one (essentially obtainable by swapping the kinematics of the above process, $x_{1} \leftrightarrow x_{2}, r_{1} \leftrightarrow r_{2}$ ) one may guess at the radiation pattern for the $1 \rightarrow 3+\overline{3}$ and $8 \rightarrow 3+\overline{3}$ processes:

$$
\begin{aligned}
& f_{1 \rightarrow 3+\overline{3}}\left(\theta_{13}\right)=f_{1}\left(\theta_{13}\right)+f_{2}\left(\theta_{13}\right)+2 \sqrt{f_{1}\left(\theta_{13}\right) f_{2}\left(\theta_{13}\right)} \\
& f_{8 \rightarrow 3+\overline{3}}\left(\theta_{13}\right)=f_{1}\left(\theta_{13}\right)+f_{2}\left(\theta_{13}\right)-\frac{2}{9} \sqrt{f_{1}\left(\theta_{13}\right) f_{2}\left(\theta_{13}\right)} .
\end{aligned}
$$

This turns out to be a good approximation up to fairly large $x_{3}$ values, i.e. so long as the spin structure is not too important, see e.g. Fig. 4 . The importance of the interference term also gives a simple explanation for the difference between the height of the peaks in the full and dashed curves in Fig. $3 \mathrm{a}-\mathrm{b}$.

\subsection{Parity dependence}

Let us further quantify differences induced by having or not a $\gamma_{5}$ factor in the matrix element, i.e. between vector $(V)$ and axial vector $(A)$ sources, between scalar $(S)$ and pseudoscalar $(P)$ ones, etc. We will use a measure which is the mean of the matrix element ratios over the whole phase space for gluon emission. Since the matrix elements with and without the $\gamma_{5}$ coupling both have the same divergence structures the ratio is well-behaved everywhere. All phase space points are here given equal weight, so large differences may not always be reflected in significant changes of the radiation pattern. To be specific, we study the function

$$
\langle Q\rangle\left(r_{1}, r_{2}\right)=\frac{1}{\int \mathrm{d} x_{1} \mathrm{~d} x_{2}} \int Q\left(x_{1}, x_{2}, r_{1}, r_{2}\right) \mathrm{d} x_{1} \mathrm{~d} x_{2},
$$

where

$$
Q\left(x_{1}, x_{2}, r_{1}, r_{2}\right)=\frac{\sigma_{0}^{1}}{\sigma_{0}^{\gamma_{5}}} \frac{\mathrm{d} \sigma^{\gamma_{5}} / \mathrm{d} x_{1} \mathrm{~d} x_{2}}{\mathrm{~d} \sigma^{1} / \mathrm{d} x_{1} \mathrm{~d} x_{2}} .
$$

$Q\left(x_{1}, x_{2}, 0,0\right)$ is equal to unity in all of phase space and in most cases $Q\left(x_{1}, x_{2}, r, 0\right)$ is also equal to unity. The exception is when the $r=0$ mass is a boson ( $\operatorname{spin} 0$ or 1 ) and the $r \neq 0$ one is spin $\frac{1}{2}$. All the processes in Table 1 which have both non- $\gamma_{5}$ and $\gamma_{5}$ couplings are represented in Fig.5. Processes with the same spin structure have the same ratio, where both the spin of the initial and final states, as well as the order of the decay products, are of importance. We notice that the difference between e.g. vector and axial vector couplings can be rather large in some cases, even for small and intermediate masses. The most important case, however, with two fermions in the final state, show small differences for $r<0.5$, so this aspect will only be significant in the case of top production. Physical consequences will be investigated in Section 5 . 

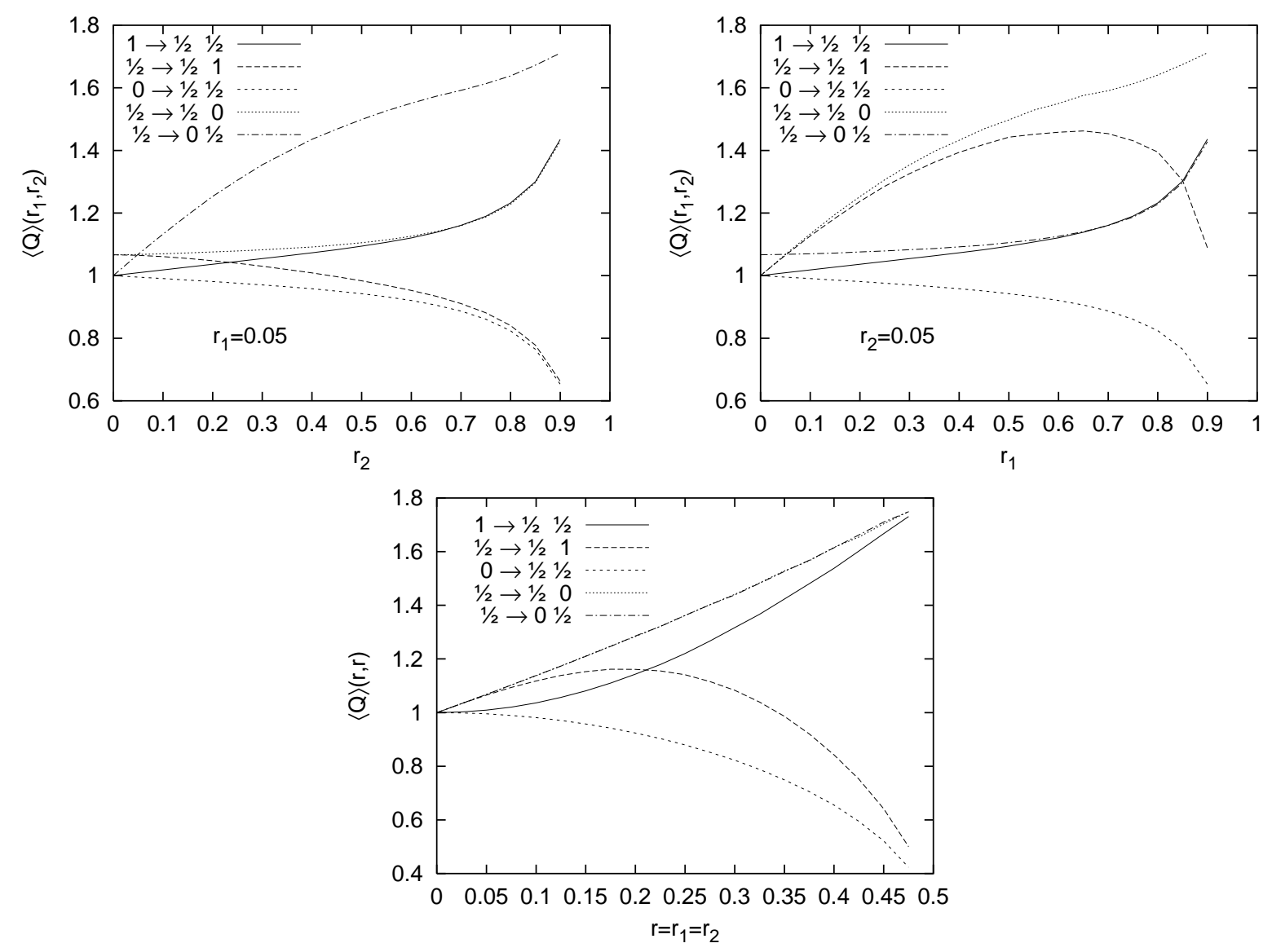

Figure 5: $\langle Q\rangle\left(r_{1}, r_{2}\right)$, eq. (51), with $r_{1}$ fixed, $r_{2}$ fixed, or $r=r_{1}=r_{2}$. The processes are grouped according to spin structure.

\section{Applications}

In this section, the matrix element corrected shower is used to study some processes at current and future colliders. It is not to be seen as a comprehensive review, but as simple examples intended to illustrate the main features.

\subsection{Bottom in $\mathrm{Z}^{0}$ decay}

We start by examining a process where data with good statistics already exists and detailed comparisons are possible, namely gluon radiation off bottom quarks produced in the process $\mathrm{e}^{+} \mathrm{e}^{-} \rightarrow \mathrm{Z}^{0} \rightarrow \mathrm{b} \overline{\mathrm{b}}$ at the $\mathrm{Z}^{0}$ pole. Gluons are not observed directly in the final state, instead they materialize as jets of hadrons. We will mainly consider jets on the parton level, but we also study the effects of hadronization and decays. The experimental data with which our results are compared have already been corrected to the parton level, using, among others, the same model for fragmentation which we will use.

Jets are constructed by considering all pairs of particles $(i, j)$ in an event, where the particles can be hadrons, partons or clusters of hadrons or partons, finding the pair with the smallest "distance" $y_{i j}$. If this number is smaller than the "jet resolution" parameter, $y_{c}$, the pair is joined into a new cluster by summing up their four-momenta. This procedure is repeated until all $y_{i j}$ are larger than $y_{c}$. The resulting clusters are the jets in the event 
at the resolution scale $y_{c}$. Jet algorithms differ mainly by the definition of $y_{i j}$, and several jet measures have been proposed in the literature [32, 35]. Following the lead of the LEP analyses we want to compare with, we have settled on the DURHAM [32] algorithm which defines

$$
y_{i j}=\frac{2 \min \left(E_{i}^{2}, E_{j}^{2}\right)\left(1-\cos \theta_{i j}\right)}{E_{\mathrm{vis}}^{2}} .
$$

For small angles, this is approximately the relative transverse momentum squared of the pair, scaled to the visible energy in the event. If jets are constructed with a large $y_{c}$, only the most energetic partons will be resolved, and most events will become 2-jet events. When $y_{c}$ is decreased, smaller structures will start to emerge and multi-parton final states become more important. It is in this region that the parton shower approach to gluon radiation is most useful.

For a primary quark flavour q, the $n$-jet rate is defined as

$$
R_{n}^{q}\left(y_{c}\right)=\frac{\sigma_{\mathrm{q} \overline{\mathrm{q}} \rightarrow n \text { jets }}\left(y_{c}\right)}{\sigma_{\mathrm{q} \overline{\mathrm{q}} \rightarrow \text { hadrons }}}
$$

which for $n>2$ is generally a decreasing function of $y_{c}$. We want to study the difference between gluon radiation off $\mathrm{b}$ quarks and off light quarks. A suitable observable is then the ratio between the respective n-jet rates

$$
R_{n}^{b l}\left(y_{c}\right)=\frac{R_{n}^{b}\left(y_{c}\right)}{R_{n}^{l}\left(y_{c}\right)}, \text { with } l=\mathrm{u}, \mathrm{d} \text { or s. }
$$

Experiments at LEP [36, 37] have found both $R_{3}^{b l}$ and $R_{4}^{b l}$ to be smaller than one, approaching unity for large $y_{c}$. This can be understood qualitatively as a consequence of the well-known dead cone effect [4], stating that the radiation of collinear gluons off heavy quarks is suppressed. The production of well separated jets, however, is not significantly suppressed and should approach that of the light quarks for large $y_{c}$.

We want to study this effect more quantitatively by using our improved, matrix element corrected, parton shower. The new approach, described in Section 3, has been implemented in Pyтніа 6.153. This is compared to PүтніA 6.152 containing the older approach of Section 2.4, which includes the correct massive matrix element correction in the first emission only. As a reference, we also include results from the algorithm implemented in PyтніA 6.129, where mass-effects are incorrectly included in the matrix element correction of the first emission, cf. Section 2.3.

The jet rate reflects both the amount of energy radiated and the direction in which it is radiated. Fig. 6a shows the gluon radiation pattern in the process $\mathrm{e}^{+} \mathrm{e}^{-} \rightarrow \mathrm{Z}^{0} \rightarrow \mathrm{q} \overline{\mathrm{q}}$ at the $\mathrm{Z}^{0}$-pole, for the old and the new shower routines both for light ( $\mathrm{u}, \mathrm{d}$ and $\mathrm{s}$ ) quarks and heavy (bottom) quarks. The angle, $\theta_{g}$, is defined as the angle between the radiated gluon and the primary quark in the CM system of the primary quark pair. In principle, gluons are radiated by the $q \bar{q}$ dipole as a whole, but in our implementation each radiated gluon is assigned to an initial quark, and in the collinear limit this separation is quite sensible. In the case of the total energy flow, Fig. 6b, we add the two contributions, and include the energy taken by quarks produced in gluon splitting, so two symmetrical peaks appear. Fig. 6 $6 \mathrm{c}$ shows the distribution of $\Delta E=E_{\mathrm{rad}} / E_{\mathrm{CM}}$, i.e. the total energy fraction radiated in the shower. Fig. 6d, finally, shows the gluon multiplicity in the shower, which obviously is quite dependent on the lower shower cut-off $Q_{0}$.

We note first of all that the difference between light and heavy quarks is largest in the region of small angles, the dead cone, and also that the radiated energy fraction is 

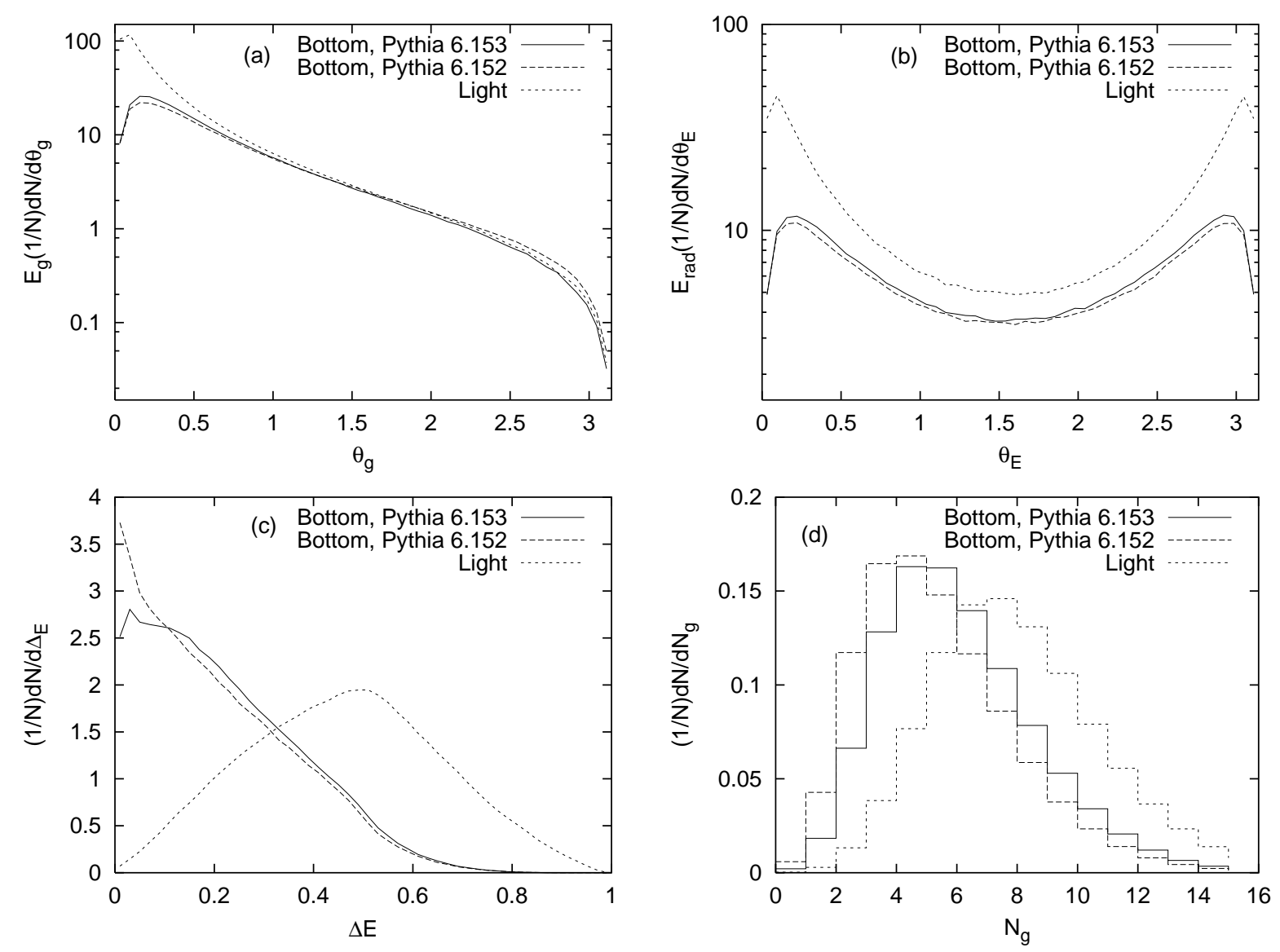

Figure 6: Gluon radiation and energy flow in the process $\mathrm{e}^{+} \mathrm{e}^{-} \rightarrow \mathrm{Z}^{0} \rightarrow \mathrm{q} \overline{\mathrm{q}}$, where $\mathrm{q}$ is either a light $(\mathrm{u}, \mathrm{d}, \mathrm{s})$ quark or a b quark. The b-mass is here set to the default in Pythia, $4.8 \mathrm{GeV}$. (a) Energy weighted gluon angle distribution. (b) Energy flow. (c) Radiated energy fraction, $\Delta E=E_{\mathrm{rad}} / E_{\mathrm{CM}}$. (d) Gluon multiplicity.

much larger for light quarks, peaked around 0.5 if $Q_{0}=1 \mathrm{GeV}$ is used to remove the collinear emission off light quarks. For heavy quarks, this collinear emission is regulated by the mass. For large angles, the light and heavy quark distributions converge as they should. In the new approach, the amount of gluon radiation in b events at small angles is somewhat enhanced relative to the old one, where the dead cone was slightly exaggerated, but differences are small. By the change of evolution variable, eq. (26), the fraction of events without any shower at all $(\Delta E=0)$ has decreased by almost a factor of 3 from about 4 per mille to 1.4. As a consequence, the peak at $\Delta E=0$ has been flattened out. The gluon multiplicity has also increased slightly.

We now want to investigate how the changes in the algorithm affect $R_{3}^{b l}$ and $R_{4}^{b l}$. Since one is studying small deviations from unity, these measures are very sensitive to changes in the gluon radiation pattern. Also, the LEP experiments have large samples of $Z^{0}$ events and studies at the per cent level are feasible. We will mostly study the behaviour of the model on the parton level, comparing different alternatives. Here the parton level is defined by the partonic configuration at the shower cut-off scale $Q_{0}$, below which no further emissions occur. But first we should comment on the effects of fragmentation. Below the $Q_{0}$ scale, the Lund string fragmentation model [1] describes how the partons transform into the primary hadrons. Subsequently these may decay further. Once a $Q_{0}$ 

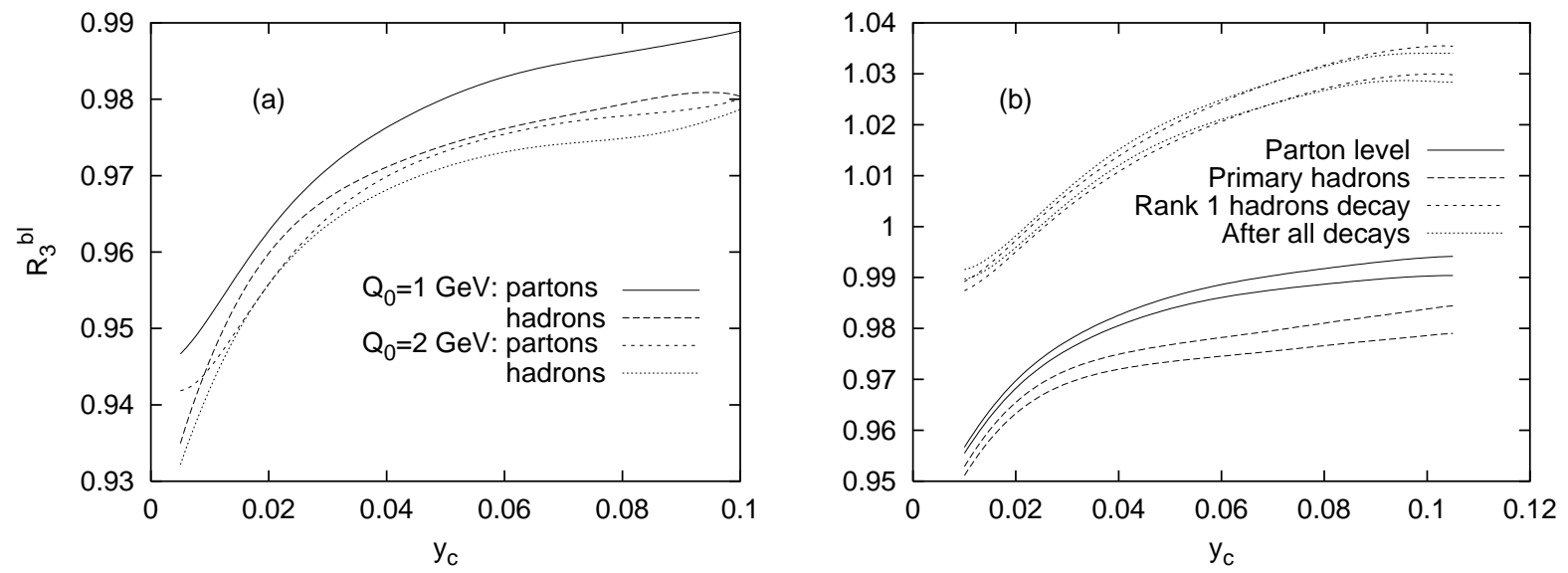

Figure 7: Study of fragmentation and decay effects. (a) Effects of changing the cut-off, $Q_{0}$, in the shower, both on the parton level and at the level of primary hadrons (i.e. before decays). (b) Effects of hadronization, decay of rank 1 hadrons and decay of all hadrons. In (b) each alternative is represented by the $\pm 1 \sigma$ curves given by the Monte Carlo statistics.

has been chosen, the parameters of the fragmentation model should be fitted to data. Here we will only study the variation with $Q_{0}$, both at the parton level and the hadron level, without any such retuning. At the hadron level, we consider first the primary hadrons and study decay effects separately.

Fig. 7 a shows the effect of fragmentation and the $Q_{0}$ dependence. We calculate $R_{3}^{b l}\left(y_{c}\right)$ at the parton level and at the level of primary hadrons for $Q_{0}=1$ and $2 \mathrm{GeV}$, respectively. The $Q_{0}$ dependence is largest on the parton level with $R_{3}^{b l}$ slightly lower for the larger $Q_{0}$. This is because the increase in $Q_{0}$ increases the 3 -jet rate for light quark events, while heavy-quark events are not influenced as much: collinear emissions, which dominate at the end of the cascade, are suppressed anyway. Intuitively one might have expected the jet rate to be higher for a lower $Q_{0}$, since this corresponds to a larger number of partons, but the further partons emitted between 1 and $2 \mathrm{GeV}$ cannot give rise to new jets of their own. They only smear out the energy of the existing jets and possibly make them fall below the cut-off. Furthermore, the hadronization stage tends to decrease the $Q_{0}$ dependence because the Lund model is infrared safe and collinear gluon emissions do not affect the string fragmentation process. A retuning of the fragmentation parameters would further limit the effect of small changes in $Q_{0}$.

Fig. $7 \mathrm{~b}$ shows the effects of fragmentation and decay. The rank 1 hadrons are the ones that contain the primary quarks from the decay of the $\mathrm{Z}^{0}$. In the case of bottom production, the rank 1 hadrons will be mainly $\mathrm{B}$ mesons and $\Lambda_{\mathrm{b}}$ baryons. Because of the hard fragmentation function for heavy hadrons, the multiplicity of primary hadrons in $\mathrm{b}$ events is smaller than that in a light quark event, reflected in the lower $R_{3}^{b l}$ curve. However, once the heavy hadrons have decayed, their decay products will more than compensate this. Thus, if the primary hadrons are allowed to decay, the value of $R_{3}^{b l}$ lies above unity for $y_{c}>0.03$. So, on the one hand, heavy hadrons take a large part of the primary quark energy, but they also decay to many particles. For $R_{3}^{b l}$ there is not a large difference between allowing all primary hadrons to decay or only the rank 1 ones, which can be seen in the small difference between the two top curves in Fig. $7 \mathrm{~b}$. In the following, we will compare our results to experimental data on the parton level, bearing in mind 

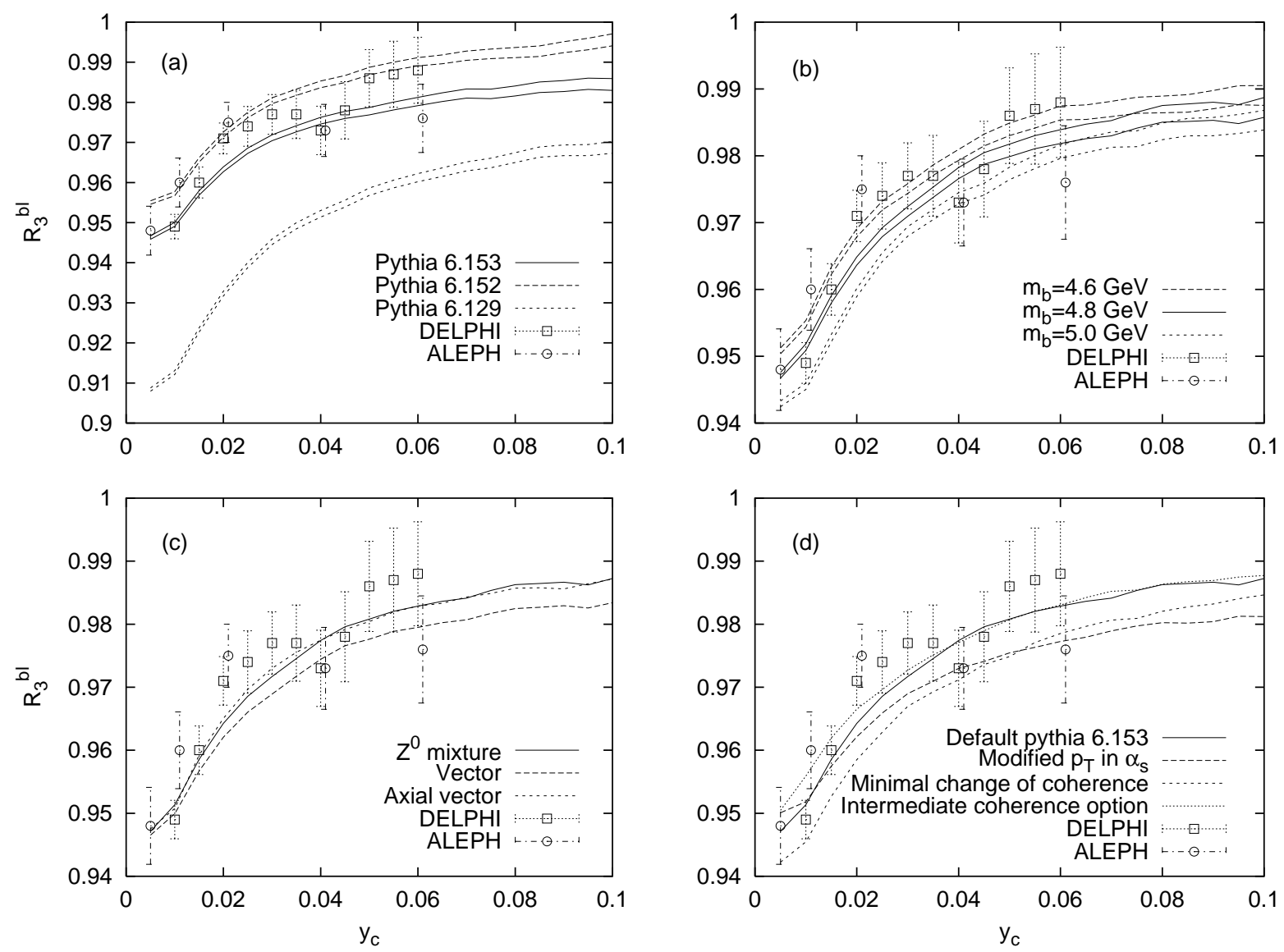

Figure 8: $R_{3}^{b l}\left(y_{c}\right)$ for different model parameters and variations. (a) Different main versions. Some variations of the latest version (6.153): (b) Different bottom masses. (c) Different sources. (d) Other minor variations of the main theme, see the text for details. The data are from [36] and [37]. The data points from AlEPH (except the first) have been shifted .001 units in $y_{c}$ for clarity. In (a) and (b) the $\pm 1 \sigma$ curves are given, while only the central value is shown in (c) and (d) for clarity, but the statistics is comparable.

that this comparison can be ambiguous in view of the dependence on $Q_{0}$. Our aim here is not to achieve a perfect fit to data, but merely ensure that the results are at the right level. As we will see, the data is anyway not good enough to make precise discriminations between all different model variations.

In Figs. 8 9, $R_{3}^{b l}$ and $R_{4}^{b l}$ are shown as functions of $y_{c}$ for several model variations. Figs. Ba, 9a show the difference between the old and the modified shower models. An even older version, where mass effects are exaggerated, is also shown as reference. Each model curve is displayed as a one sigma band, showing the size of the Monte Carlo error for $15 \cdot 10^{6}$ events of each kind. From the increase in multiplicity and energy flow in b events in the new model, one would naively expect the 3- and 4-jet rates to increase for heavy quarks, thus increasing $R_{n}^{b l}$. Actually, the main effect is to reduce the 3 -jet rate for heavy quarks, again illustrating that allowing more collinear/soft emissions will not necessarily increase the rate of well separated jets. The naively expected increase of $R_{4}^{b l}$ (from subsequent branchings) is balanced by a corresponding "smearing" loss as for $R_{3}^{b l}$, giving only a small net effect.

As already noted, the old and new models differ mainly in the treatment of subsequent 

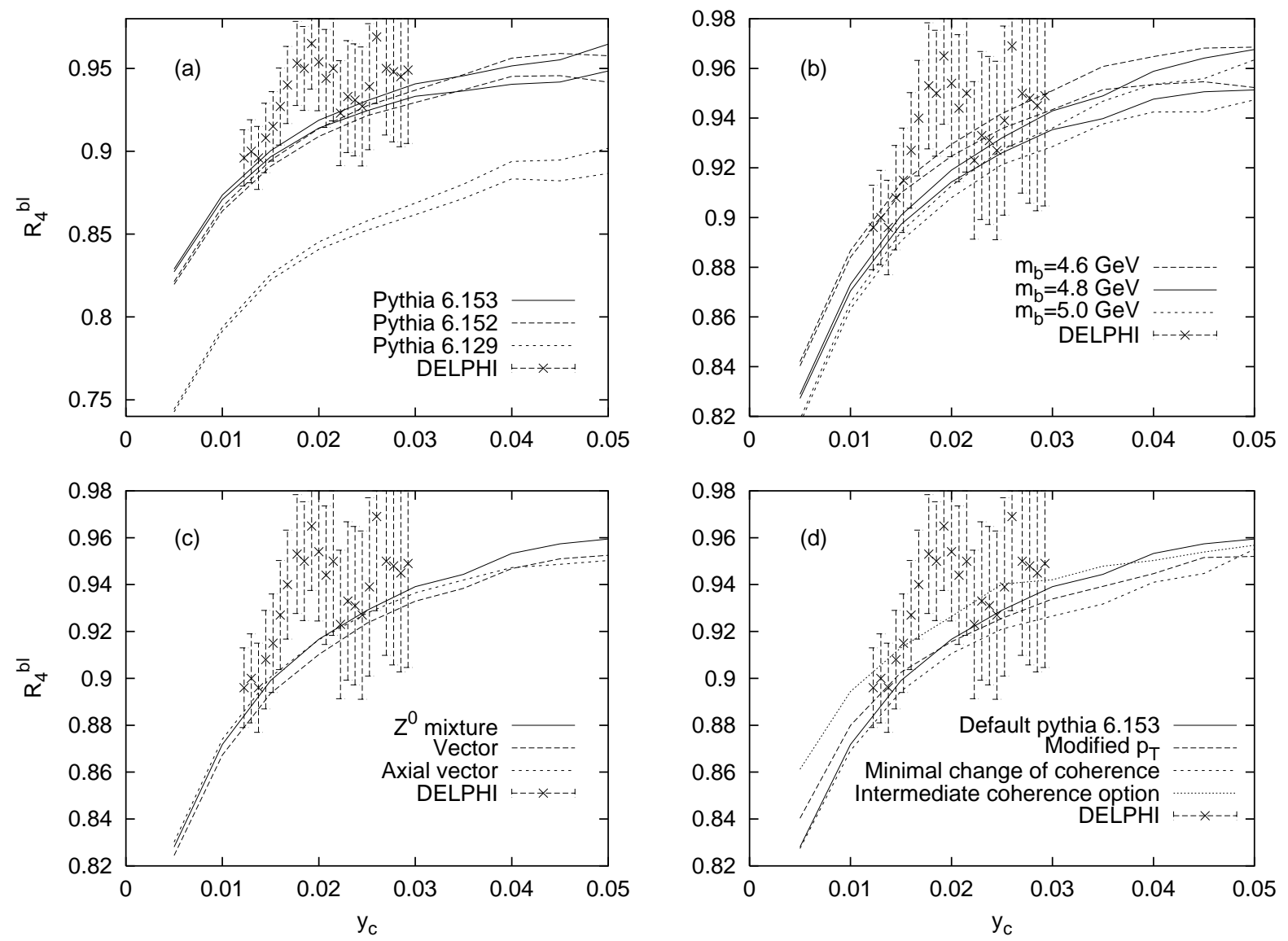

Figure 9: $R_{4}^{b l}\left(y_{c}\right)$ for different model parameters and variations. See caption Fig. 8 for details.

branchings; both include matrix element corrections to the first gluon emission on each side of the event. A clear discrimination between these two versions is not possible, especially in view of the variations that follow below and the relatively large experimental errors. It is clear, however, that the algorithm in PYTHIA 5.129 is ruled out by the data.

Next we consider variations to the new default, in order to assess the uncertainties of the model:

- The bottom mass.

- Parity dependence (vector vs axial vector source).

- Change of $p_{\perp}^{2}$ argument in $\alpha_{\mathrm{s}}$ from $z(1-z) m_{a}^{2}$ to $z(1-z) m_{a}^{2}\left(1-m_{b}^{2} / m_{a}^{2}\right)^{2}$ in a branching $a \rightarrow b c$, with $a$ being a heavy quark.

- Coherence effects.

The main free parameter is the bottom mass, and Figs. $8 \mathrm{~b}, 9 \mathrm{~b}$ show the result of varying this mass between $4.6 \mathrm{GeV}$ and $5 \mathrm{GeV}$. Fits of NLO QCD calculations to LEP data give a value around $3 \mathrm{GeV}$ for the running bottom mass in the $\overline{\mathrm{MS}}$ renormalization scheme [5, 36, 37] at renormalization scale $m_{\mathrm{Z}}$. Our model is based on LO matrix elements and the mass in our case is the constituent quark mass, so they need not agree. However, a somewhat lower mass than the default seems to be favoured, especially for $R_{4}^{b l}$.

In sections 4.2 and 4.3 we saw that there are slight differences between different sources. The relevant mixture for $\mathrm{Z}^{0}$ is given by eq. (42), but in Figs. 8c, 9c we study the two extreme cases of a pure vector source and a pure axial vector one. At the $\mathrm{Z}^{0}$-pole, the 
axial vector component dominates, as can be seen in the figure, but at higher energies the vector one will take over. We see that the differences are non-negligible, but not as large as the shown mass dependence, at least for small $y_{c}$.

Finally, in Figs. 8d, 9d, some further aspects of the model, discussed in Section 3.4, are varied. Both the modified $p_{\perp}$ in $\alpha_{\mathrm{s}}$ and the "minimal" change of coherence tends to reduce $R_{3}^{b l}$, again showing how an increase in the total amount of radiation need not give more separate jets. The "intermediate" coherence option, which still is a realistic alternative, does give an increase, especially of $R_{4}^{b l}$. Thereby the overall agreement with data is improved, as much as with a reduced b mass, although uncertainties are sufficiently large that no firm conclusions should be drawn.

Other minor issues could be studied and here we just mention a few. The definition of $R_{n}^{b l}$ used here is based on a classification of events with heavy or light primary quarks. An alternative is to use a ratio between b-tagged and anti b-tagged events. The difference here is in the classification of events with gluon splitting, $\mathrm{g} \rightarrow \mathrm{b} \overline{\mathrm{b}}$, in the shower. For the $R_{3}^{b l}$ ratio this is a minor issue, but it is non-negligible in the case of $R_{4}^{b l}$ [5]. This uncertainty is already included in the error-bars for the experimental points in Fig. 9, so need not be studied separately here. Another issue is the energy dependence of the results. Obviously the mass effects will decrease for larger energies, because of the decrease of $r=m_{\mathrm{b}} / \sqrt{s}$, and this has already been studied at $189 \mathrm{GeV}$ [5]. At the same time, however, the fraction of vector coupling increases significantly above the $\mathrm{Z}^{0}$-pole, thus decreasing $R_{3}^{b l}$, cf. Fig. \& $\mathrm{c}$. At intermediate energies this could therefore give rise to a partial cancellation of effects.

\subsection{Bottom in Higgs decay}

We now turn to gluon radiation in Higgs decay. In the standard model, the decay $\mathrm{H}^{0} \rightarrow \mathrm{b} \overline{\mathrm{b}}$ is expected to be large, or even dominate, for Higgs masses up to the $\mathrm{W}^{+} \mathrm{W}^{-}$threshold, and in extensions to the standard model also heavier Higgs states can have significant $\mathrm{b} \overline{\mathrm{b}}$ branching ratios. When the fragmentation function for $\mathrm{B}$ mesons, i.e. the distribution of $z=2 E_{\mathrm{B}} / \sqrt{s}$, is measured at LEP1, the $\mathrm{b}$ quark is produced from a spin 1 source. If instead the source is a Higgs boson, the difference in gluon radiation could give rise to a changed fragmentation function. Such a change would influence the experimental vertex detection efficiency which, if uncorrected, would give rise to errors in the determination of cross sections. It is therefore important to be able to describe in detail the gluon radiation pattern in this decay. A different measure of the nature of the source is the jet topology, which is also studied in the form of a modified $R_{3}^{b l}$ ratio, cf. the preceding section.

We choose to study the production of b $\bar{b}$ pairs at a CM energy of $130 \mathrm{GeV}$. This number is in the middle between the current lower limit and the $\mathrm{W}^{+} \mathrm{W}^{-}$threshold, at around the value expected for the MSSM $h^{0}$, but obviously the relative comparison of the sources is only mildly energy-dependent. Five different sources are compared. The first sample is considered as a reference and consists of gluon radiation in the decay $\gamma^{*} / Z^{*} \rightarrow q \bar{q}$, where $\mathrm{q}$ is a light $\mathrm{u}, \mathrm{d}$ or $\mathrm{s}$ quark. For simplicity they are assumed to be produced in equal amounts. The other four samples consist of gluon radiation in the decay $X \rightarrow \mathrm{b} \bar{b}$, where $X$ is a vector, axial vector, scalar or pseudoscalar source. Clearly the $\gamma^{*} / \mathrm{Z}^{*}$ is a mixture, eq. (42), and also $\mathrm{h}^{0} / \mathrm{H}^{0} / \mathrm{A}^{0}$ need not be pure states, but the separation allows us to study the extreme range of possibilities.

Fig. 10a shows the angular energy flow. The difference between having or not having a $\gamma_{5}$ in the coupling is negligible, but the radiation at large angles is larger for the spin 0 source than a spin 1 one. The measure in Fig. 10 b, i.e. the normalized 3-jet rate, is most 

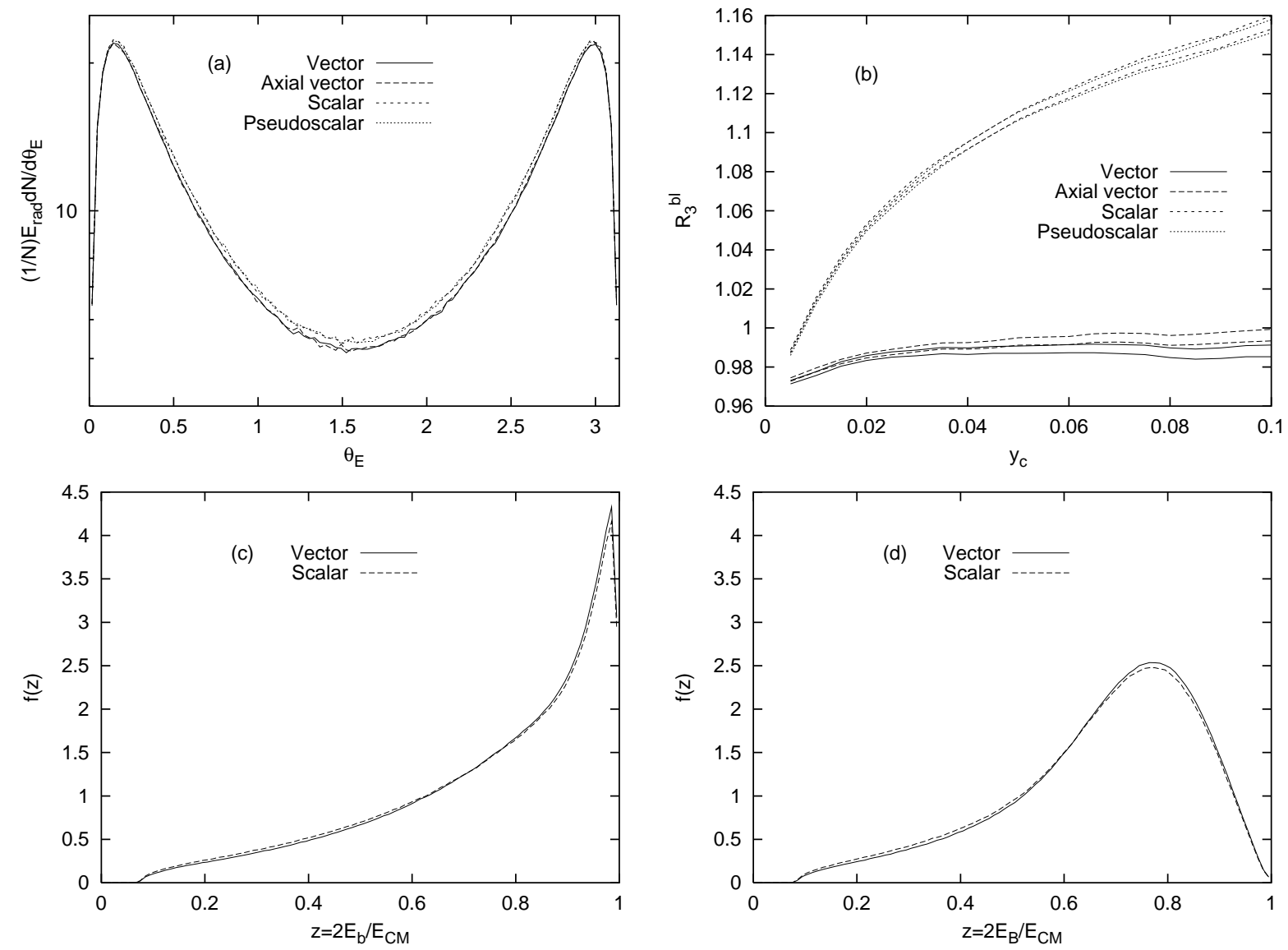

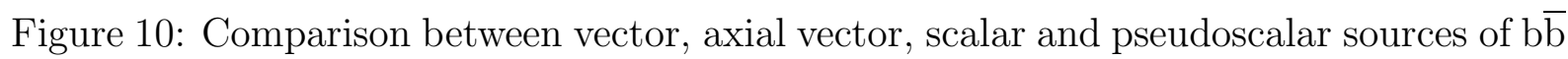
pairs at $130 \mathrm{GeV}$. (a) Energy flow in the shower. (b) 3 -jet rate, normalized to $\gamma^{*} / \mathrm{Z}^{*} \rightarrow \mathrm{q} \overline{\mathrm{q}}$, i.e. $R_{3}^{b l}\left(y_{c}\right)=R_{3}^{b}(X \rightarrow \mathrm{b} \overline{\mathrm{b}}) / R_{3}^{l}\left(\gamma^{*} / \mathrm{Z}^{*} \rightarrow \mathrm{q} \overline{\mathrm{q}}\right)$, where $X$ is $V, A, S$ or $P$ and $\mathrm{q}$ is a light flavour. $1 \sigma$ Monte Carlo error bands are shown. (c) Distribution of $z_{\mathrm{b}}=2 E_{\mathrm{b}} / \sqrt{s}$ at the parton level. (d) Distribution of $z_{\mathrm{B}}=2 E_{\mathrm{B}} / \sqrt{s}$, i.e. the fragmentation function, at the hadron level (only primary hadrons are considered).

sensitive and shows that the 3-jet rate is significantly larger for a spin 0 source, especially for well separated jets (large $y_{c}$ ), which is consistent with the larger energy flow at large angles. The parity dependence is very small, on the other hand, and is neglected in the following.

Considering the large spin dependence one could expect the fragmentation function also to change considerably when going from a vector to a scalar source. Fortunately, as can be seen in Figs. 10c-d, the changes are minor, indicating that the fragmentation function is mainly sensitive to the bulk of gluons at smaller angles, where the sources give the same emission rate. The larger gluon energy flow for a scalar source is reflected in a slightly smaller $\langle z\rangle$, with a difference of about $1 \%$ both at the parton and hadron level. The small effect on the fragmentation function is positive from an experimentalist's point of view, in that fragmentation functions measured at the $\mathrm{Z}^{0}$ pole can be simply extrapolated also for possible spin 0 sources at higher energies. 

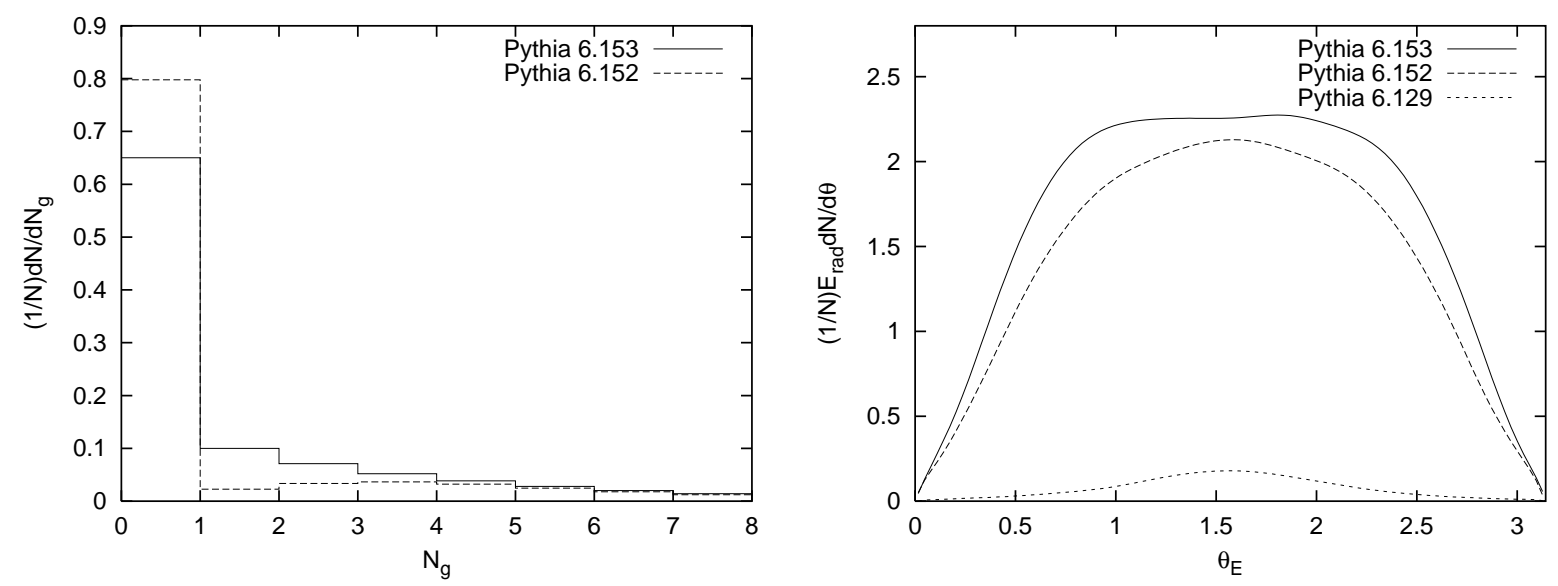

Figure 11: Gluon multiplicity and energy flow in the tॄ̄ shower at $500 \mathrm{GeV}$ centre-of-mass.

\subsection{Top production and decay}

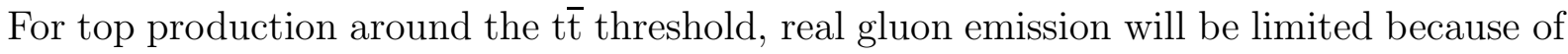
phase space, so aspects such as the form of the QCD confinement potential, best tackled with other perturbative techniques, will be more relevant [38. In this section we study the process $\mathrm{e}^{+} \mathrm{e}^{-} \rightarrow \mathrm{t} \overline{\mathrm{t}}$ at $500 \mathrm{GeV}$, where gluon emission will start to become important, and compare the new shower routine with the older versions. We focus on fundamental aspects such as gluon radiation patterns and top mass determinations. In the process we will also study gluon radiation in the decay of the top, and differences in the radiation patterns for the two decay channels $\mathrm{t} \rightarrow \mathrm{bW}^{+}$and $\mathrm{t} \rightarrow \mathrm{bH}^{+}$to demonstrate the advertised process dependence of the new shower routine.

We first study gluon radiation in the production of the top. The matrix element used in the correction of the shower is an energy dependent mixture of vector and axial vector ones. High above the $\mathrm{Z}^{0}$ pole, the vector part will dominate and at $500 \mathrm{GeV}$ the $\alpha$ parameter, introduced in Section 4.1 to parameterize the relative mixture, is about 0.78 for $t \bar{t}$ production. The dead cone effect is expected to be very pronounced in this case, because the scaled mass is large, around $r=0.35$. Fig. 11 shows the gluon multiplicity and

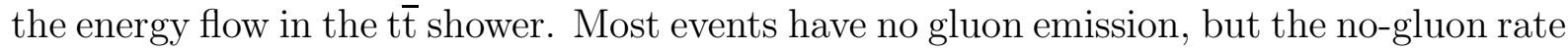
has decreased in the new shower as expected. The result of an even older version, where the dead cone effect is severely exaggerated in all branchings, is shown as a reference. The lesson is that the correct description of the first emission, i.e. the difference between 6.129 and 6.152, is much more important than that of subsequent emissions, between 6.152 and 6.153. Once a gluon is emitted, it tends to be quite energetic and splits into several further gluons. This is the reason for the large spread in the multiplicity and the dip in this distribution for the old shower routine. The total energy flow has also increased in the new shower relative to the older ones. Even if the energy flow seems to be large at large angles, most energy is kept by the original top quarks at $\theta_{\mathrm{E}}=0$ and $\pi$ respectively.

The dominant top decay is $\mathrm{t} \rightarrow \mathrm{bW}^{+}$, where further gluons will be radiated. In the old shower routine, gluon radiation off the $b$ did not take into account the full $t-b$ interference structure. Furthermore, gluon radiation in a hypothetical decay $\mathrm{t} \rightarrow \mathrm{bH}^{+}$was the same as in the one above, apart from the difference induced by the different $\mathrm{W}$ boson and Higgs masses. In order to separate off this trivial mass effect we put $m_{\mathrm{H}}=m_{\mathrm{W}}$, well aware that this is not realistic. 

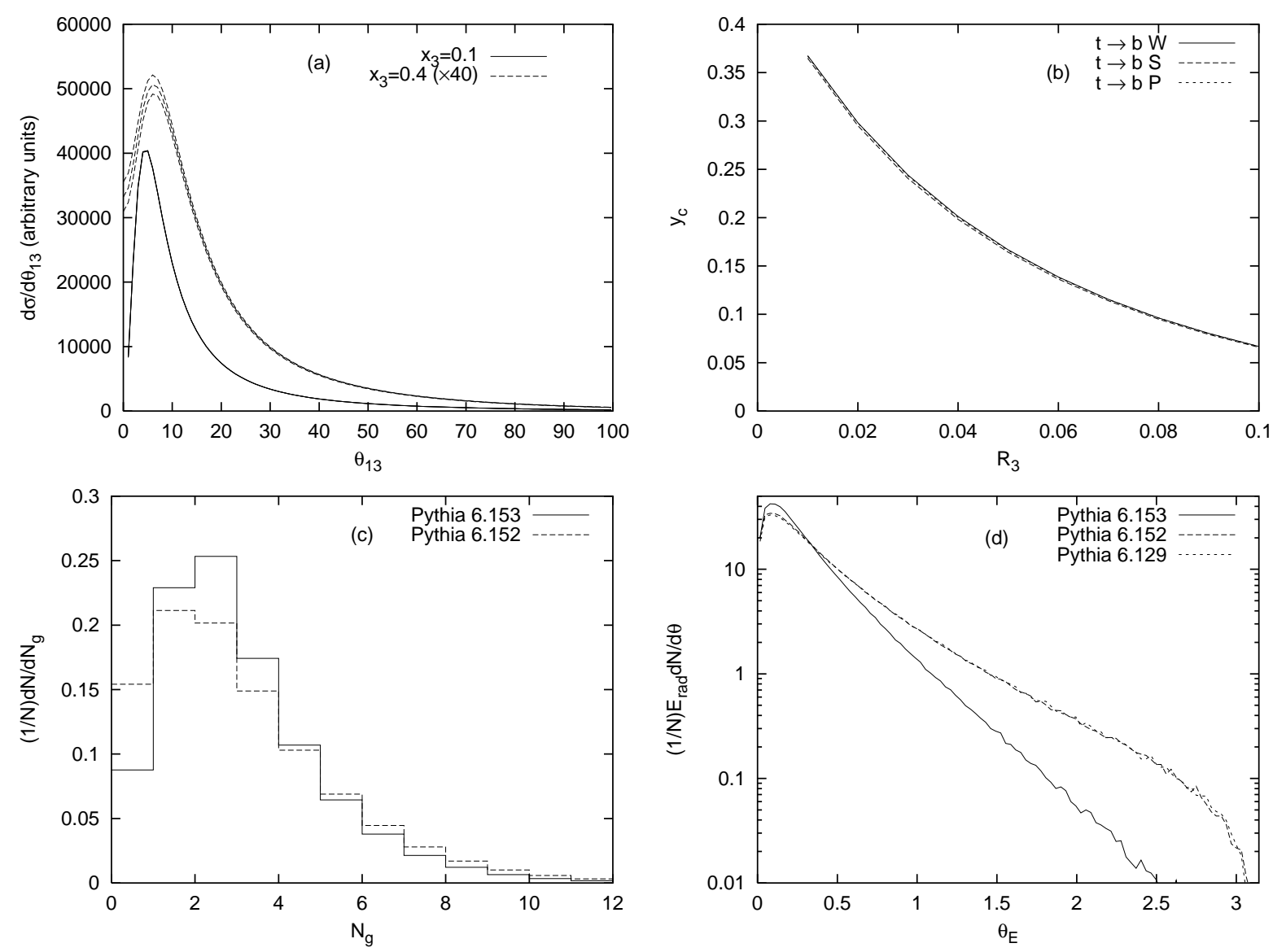

Figure 12: (a) The gluon emission rate as a function of the emission angle $\theta_{13}$ for top decay, $\mathrm{t} \rightarrow \mathrm{b} X$. The emission rate is given for the two gluon energies $x_{3}=0.1$ (full curves) and $x_{3}=0.4$ (dashed). The curves corresponding to the higher energy have been multiplied by a factor of 40 for clarity. Three different curves are shown for each energy: pseudoscalar Higgs, W boson and scalar Higgs (from high to low curves). (b) The 3-jet rate in complete $t \bar{t}$ events as a function of $y_{c}$ for the three different cases above, where the $\mathrm{W} / \mathrm{H}$ are removed from the cluster search. (c-d) Gluon multiplicity and energy flow in the shower induced by the top decay (in the rest-frame of the decaying top with the $\mathrm{W}$ at $\left.\theta_{\mathrm{E}}=\pi\right)$.

The matrix element used in the decay $\mathrm{t} \rightarrow \mathrm{bW}^{+}$has the familiar $V-A$ coupling, but the $\mathrm{t} \rightarrow \mathrm{bH}^{+}$case is less obvious. In the MSSM the vertex factor is proportional to $m_{\mathrm{t}}\left(1-\gamma_{5}\right)+m_{\mathrm{b}} \tan ^{2} \beta\left(1+\gamma_{5}\right)$, i.e. a parameter-dependent mixture of scalar $(S)$ and pseudoscalar $(P)$ couplings. Naively, the $S-P$ one would be expected to dominate because of the large difference between the top and bottom masses. However, the favoured values of $\tan \beta \gtrsim 3$ could cancel this effect and give rise to an almost pure scalar coupling. We therefore study the two extreme cases of $S$ and $P$ separately. The difference between the matrix elements of this type has been studied in Tables 2 and 3 and found to be non-negligible for massive daughters but to vanish in the massless limit. For the process $\mathrm{t} \rightarrow \mathrm{bX}$, where $X$ is a $\mathrm{W}^{+}$, pure scalar $\mathrm{H}^{+}$or pure pseudoscalar $\mathrm{H}^{+}$, some differences are visible in the gluon energy flow for larger gluon energies, see Fig. 12a, but the differences induced in observables such as the jet rates are small, Fig. 12b. That is, we are closer to the massless case of Table 3 , indicating that the small mass of the radiating $\mathrm{b}$ is more 

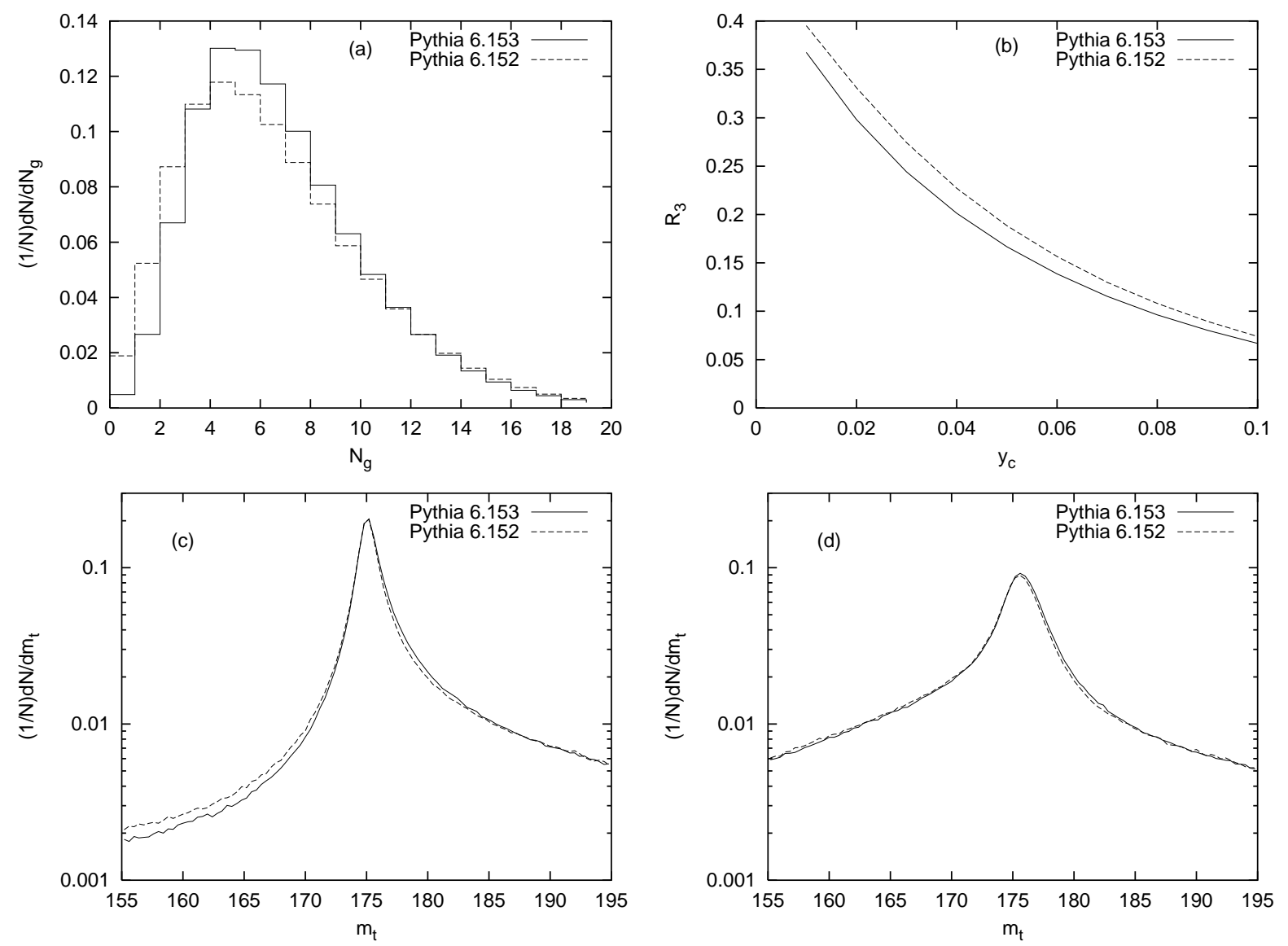

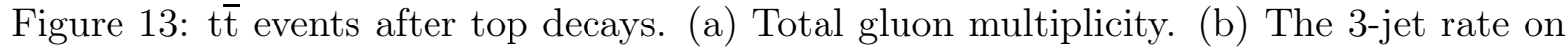
the parton level, with $\mathrm{W} / \mathrm{H}$ removed from the clustering. Initial-state photon radiation is included, but both models are equally affected by this. (c) Reconstructed top mass, parton level. (d) Reconstructed top mass, hadron level.

important than the large mass of the non-radiating $\mathrm{W}^{+} / \mathrm{H}^{+}$. In what follows, therefore, only the decay to $\mathrm{W}^{+}$is shown. This is the most important case and, as it turns out, the intermediate alternative, cf. Fig. 12a.

Figs. 12 $\mathrm{c}-\mathrm{d}$ show the gluon multiplicity and energy flow in the decay of the top for the new and the older shower routines. The gluon multiplicity is slightly more peaked in the new shower and the no-gluon rate has been somewhat reduced. The angle $\theta_{E}$ is defined as the angle of emitted energy to the primary bottom quark in the rest frame of the decaying top, which is why no peak appears at large angles, corresponding to the direction of the colour neutral $\mathrm{W} / \mathrm{H}$. In the new correction to the shower, gluon radiation at large angles is more severely suppressed. As a consequence, there is more energy left for radiation at small ones, allowing the curves to cross there. This is an effect in addition to the influence of the new matrix element correction scheme, visible e.g. in Fig. 6a. The difference between decays to $\mathrm{W}$ and $\mathrm{H}$ are very small also here (not shown). The result of PYThia 6.129 is similar to 6.152, but the dead cone is slightly more pronounced, as noted before.

In a complete event, gluon emission from the decay and production are added, and also possible radiation in hadronic decays of the $\mathrm{W} / \mathrm{H}$ must be considered. In principle, these showers will also interfere but, as a realistic first approximation, we work in the zero 
width limit where these interferences vanish. Considering the two stages of production and decay together, but ignoring the decay products of the W/H, Fig. 13a shows the total gluon multiplicity for the new and older shower routines. Since the radiation has increased in the production and decreased in the decay, effects tend to cancel. The net result is a slight increase in the average gluon multiplicity and a somewhat smaller width. The 3-jet rate, however, has decreased, Fig. 13b, presumably because of the reduced probability to have gluon emissions at large angles in the top decay, cf. Fig. 12 d.

The total gluon multiplicity is not a very good measure of the event properties and certainly not an observable. To give a practical example where gluon radiation is important, we consider the reconstruction of the top mass in a simplified scenario, assuming that the $\mathrm{W} / \mathrm{H}$ can be completely reconstructed in order only to study the effects of gluon emission in the top production and decay. We find two jets and calculate the top mass by considering the two possible combinations of jet $+\mathrm{W}$ momenta. The combination which minimizes $\left(m_{1}-m_{\mathrm{t}}\right)^{2}+\left(m_{2}-m_{\mathrm{t}}\right)^{2}$, where $m_{t}=175 \mathrm{GeV}$, gives the reconstructed top masses $m_{1}$ and $m_{2}$. The distribution of the reconstructed mass is given in Fig. 13kc, where jets are clustered on the parton level. The reduced radiation level survives in this measure as a slightly narrower peak for the new shower. The effects of fragmentation and decay are quite large for the reconstructed mass, but some differences still survive in the high mass wing, as seen in Fig. 13jd.

\subsection{Supersymmetry production and decay}

In supersymmetric models, a whole new set of processes involving coloured particles is introduced. We have calculated the most important LO matrix elements of the MSSM, listed in Table 1, to be used as input to the parton shower routine. In versions of PyTHIA prior to 6.153, supersymmetric particles did not shower. Because of the large masses of these hypothetical particles, this is a good first approximation. However, if supersymmetric particles are discovered at the LHC or a future linear collider, detailed studies of their properties will profit from a better understanding of QCD radiation patterns.

A complication that appears in the MSSM is significant rates of three body decays involving one or several coloured particles in the initial or final states. Often several interfering diagrams with intermediate off-shell propagators contribute. An example is the decay $\tilde{\chi}^{+} \rightarrow \tilde{\chi}^{0} \mathrm{q}^{\prime} \overline{\mathrm{q}}$ with either a $\mathrm{W}$ or squark propagator. These processes do not fit into the present framework with corrections to matrix elements of the type $a \rightarrow b c g$. As a preliminary solution, the $\mathrm{q}^{\prime} \overline{\mathrm{q}}$ pair above is assumed to be produced from a $V-A$ source. A new kind of gluon radiation process in the MSSM is from four-vertices such as $\tilde{\mathrm{q}} \rightarrow \tilde{\mathrm{q}}^{\prime} \mathrm{W}^{+} \mathrm{g}$. These diagrams introduce no new divergences. The interference terms only contribute to the ordinary divergences, and the squared amplitude gives rise to a constant. An upper estimate is again given by the new shower expression, which is corrected by the full matrix element.

As a first example, consider top vs. stop production at a linear $\mathrm{e}^{+} \mathrm{e}^{-}$collider and the decay $\mathrm{t} \rightarrow \mathrm{bW}^{+}$vs. $\tilde{\mathrm{t}} \rightarrow \mathrm{b} \tilde{\chi}^{+}$. Again, identical masses are assumed and consequently $m_{\tilde{\mathrm{t}}}=m_{\mathrm{t}}$ and $m_{\tilde{\chi}}=m_{\mathrm{W}}$ are used. As usual, the $\mathrm{t} \overline{\mathrm{t}}$ source is an energy dependent

mixture of vector and axial vector. Only a scalar coupling is possible for $Z^{0} \rightarrow \tilde{\tilde{t}}$, so no ambiguity here either. The top decay has the $V-A$ coupling, while the stop one is a parameter dependent mixture of scalar and pseudoscalar. Again we consider the two extreme cases separately. While the stop obviously decays isotropically, the current PyтніA implementation does not include top polarization information and thus 

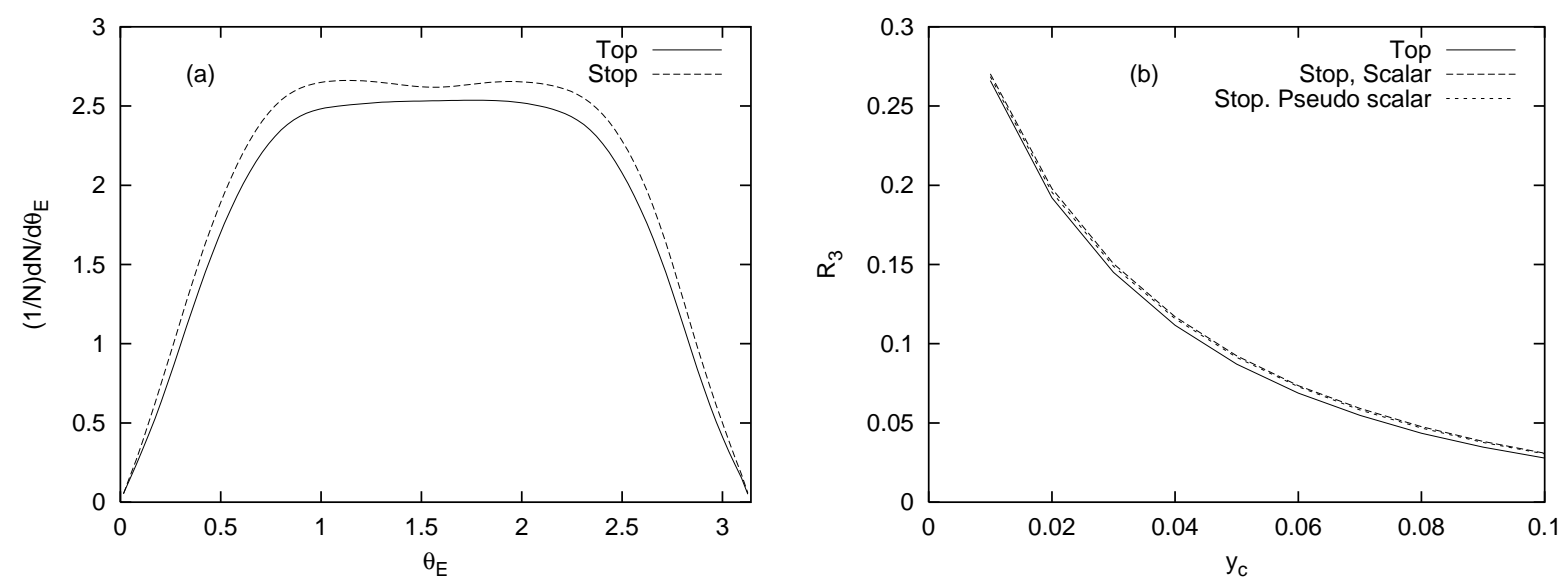

Figure 14: Top vs. stop production and decay at a $500 \mathrm{GeV} \mathrm{e}^{+} \mathrm{e}^{-}$collider. (a) energy flow in the $\mathrm{t} \overline{\mathrm{t}}(\tilde{\mathrm{t}} \overline{\mathrm{t}})$ shower. (b) 3-jet rate on the parton level for the production and decay taken together, with the $\mathrm{W} / \tilde{\chi}$ removed from the clustering.

also decays this particle isotropically. Initial-state photon radiation has not been included in the comparison, since the different threshold behaviours of the t $\bar{t} / \tilde{t} \bar{t}$ cross sections allow more energetic radiation in the former process, which will affect event topologies.

Fig. 14a shows the energy flow for top vs. stop production. The multiplicity is the same for both top and stop, but the energy flow is slightly larger for stop at large angles. The difference between the gluon radiation patterns in the top and stop decays is negligible (not shown). As a result of the increased gluonic energy flow, the 3-jet rate at the parton level, with the $\mathrm{W} / \tilde{\chi}$ removed from the cluster analysis, is slightly larger for stop, Fig. 14b. We notice further that the parity dependence is negligible also in this case. The differences between supersymmetric and standard model processes is not large in this example, at least when mass effects are neglected, and the ambiguity in the coupling structure is negligible. This is all good news, but since gluon emission from supersymmetric particles is a completely new feature, many more tests should be done.

As a last example we consider a full simulation of gluino pair production (via gg $\rightarrow$ $\tilde{g} \tilde{g}$ ) at a $14 \mathrm{TeV}$ pp collider. Since we have not developed a full shower formalism for $2 \rightarrow 2$ processes, as a first approximation the eikonal expression will be used for radiation off the $\tilde{g} \tilde{g}$ system, including a colour factor rescaling by $N_{C} / C_{F}=9 / 4$. Initial state radiation is studied separately and multiple interactions are neglected. A scenario with $m_{\tilde{\mathrm{g}}}=450 \mathrm{GeV}, m_{\tilde{\mathrm{b}}_{1}}=250 \mathrm{GeV}$ and $m_{\tilde{\mathrm{t}}_{1}}=200 \mathrm{GeV}$ is used. The dominant gluino decays are then $\tilde{\mathrm{g}} \rightarrow \tilde{\mathrm{b}}_{1} \overline{\mathrm{b}}\left(\tilde{\mathrm{t}}_{1} \overline{\mathrm{t}}\right)$ and the squarks decay by processes of the type $\tilde{\mathrm{q}} \rightarrow \tilde{\chi} \mathrm{b}$. If the $\tilde{\chi}$ is a neutralino, which here is the lightest supersymmetric particle, it will not decay further. A chargino decays to a neutralino plus either leptons or quarks in approximately equal amounts. This MSSM scenario is implemented in the PYTHIA event generator [6] and the details are described in the Spythia manual [39].

To assess the importance of gluon radiation off supersymmetric particles in a hadronhadron collision, we compare the transverse gluon energy flow from SUSY and non-SUSY particles in the final state radiation (FSR), Fig. 15a. The additional gluon radiation from coloured SUSY particles is thus seen to be small compared to the ordinary gluon radiation from quarks. In this paper we have only considered QCD final state radiation in $\mathrm{e}^{+} \mathrm{e}^{-}$annihilation. In a hadron collider environment, gluons can be radiated also from 

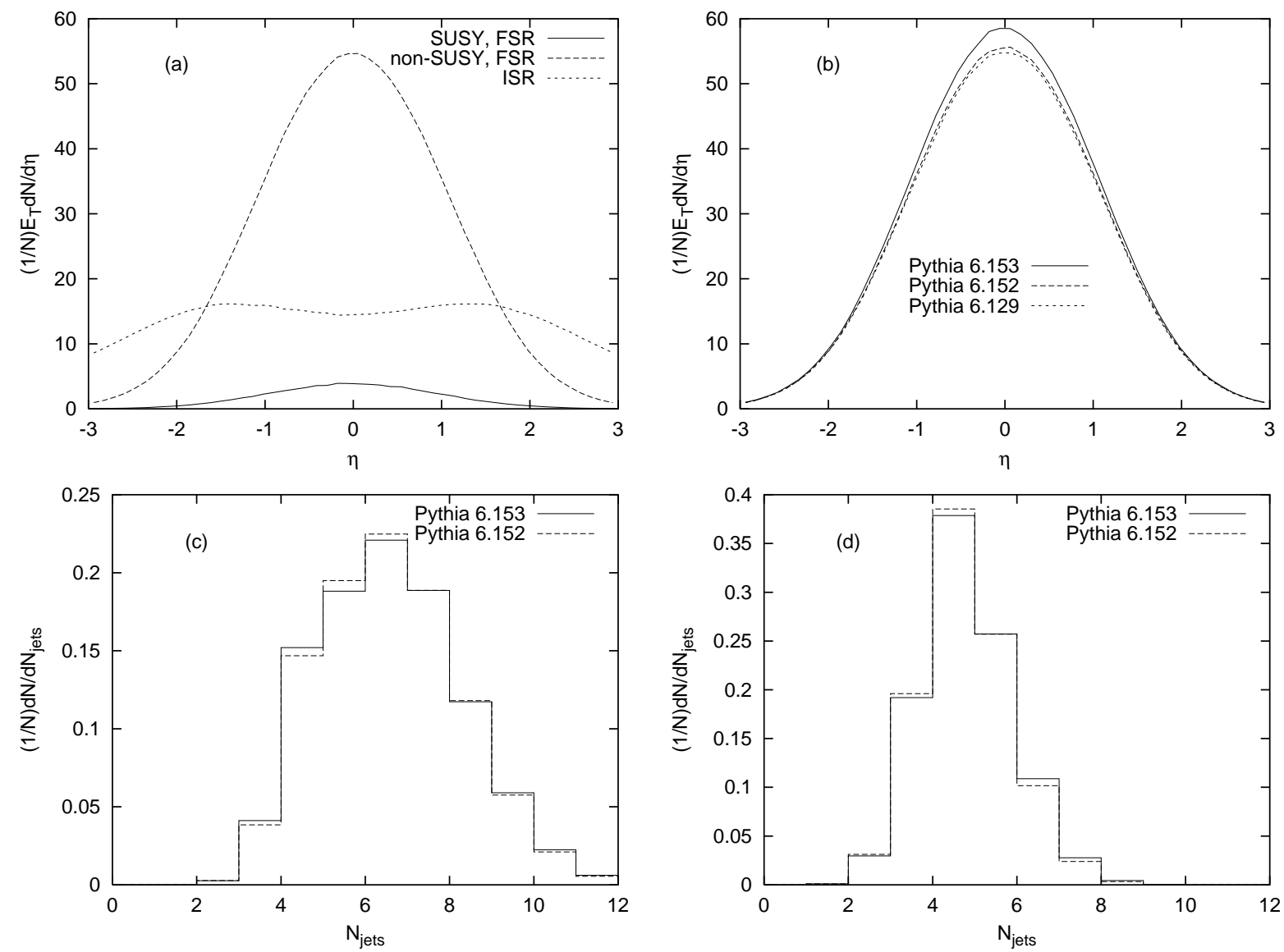

Figure 15: Gluino production at a $14 \mathrm{TeV}$ pp collider. (a-b) Transverse gluon energy flow in pseudorapidity, $\eta=\frac{1}{2} \ln \left(\left(p+p_{z}\right) /\left(p-p_{z}\right)\right)$. (c-d) Jet multiplicity for $R=0.75$, $E_{\perp, \min }=10$ and $40 \mathrm{GeV}$ respectively.

the incoming partons (initial state radiation, ISR). This contribution is almost as large as the FSR one, Fig. [15a, but the rapidity distribution is different.

In Fig. 15b the three PyтнiA versions considered in this paper are compared. Only the FSR component is shown, and the additional radiation from SUSY particles in the latest version gives rise to an increased total transverse gluon energy flow. This increase is, however, slightly compensated by the decrease in gluon radiation in the top and squark decays, cf. Section 5.3. In Figs. 15c-d the result of a simple cluster search is shown. Particles with a summed transverse energy, $\sum E_{\perp}>E_{\perp, \min }$ inside a $\sqrt{(\Delta \eta)^{2}+(\Delta \varphi)^{2}}<R$ cone are joined in a cluster. For large $E_{\perp, \min }$, the jet multiplicity is slightly increased when gluon radiation from supersymmetric particles is included. This could be caused by energetic gluons radiated off the gluinos. For small $E_{\perp, \min }$, on the other hand, smaller structures are probed and the radiation off $\mathrm{b}$ and lighter quarks is most important. In summary, we conclude that gluon radiation off supersymmetric particles at a hadron collider is small compared to the other sources, and of importance mainly in high-precision studies. 


\begin{tabular}{|c|c|c|c|c|}
\hline$\alpha_{\mathrm{s}}$ & coherence & $\mathrm{g} \rightarrow \mathrm{u} \overline{\mathrm{u}}+\mathrm{d} \overline{\mathrm{d}}+\mathrm{s} \overline{\mathrm{s}}(\%)$ & $\mathrm{g} \rightarrow \mathrm{c} \overline{\mathrm{c}}(\%)$ & $\mathrm{g} \rightarrow \mathrm{b} \overline{\mathrm{b}}(\%)$ \\
\hline$\alpha_{\mathrm{s}}\left(p_{\perp}^{2}\right)$ & full & 14.3 & 1.26 & 0.15 \\
$\alpha_{\mathrm{s}}\left(p_{\perp}^{2}\right)$ & intermediate & 14.8 & 1.27 & 0.16 \\
$\alpha_{\mathrm{s}}\left(p_{\perp}^{2}\right)$ & reduced & 21.1 & 1.92 & 0.26 \\
$\alpha_{\mathrm{s}}\left(p_{\perp}^{2}\right)$ & none & 38.8 & 3.06 & 0.31 \\
$\alpha_{\mathrm{s}}\left(m_{\mathrm{g}}^{2} / 4\right)$ & full & 12.9 & 1.15 & 0.15 \\
$\alpha_{\mathrm{s}}\left(m_{\mathrm{g}}^{2} / 4\right)$ & intermediate & 13.3 & 1.17 & 0.15 \\
$\alpha_{\mathrm{s}}\left(m_{\mathrm{g}}^{2} / 4\right)$ & reduced & 20.0 & 1.78 & 0.28 \\
$\alpha_{\mathrm{s}}\left(m_{\mathrm{g}}^{2} / 4\right)$ & none & 43.3 & 3.47 & 0.46 \\
\hline
\end{tabular}

Table 4: The rate of gluon splitting to q $\overline{\mathrm{q}}$ pairs, in $\mathrm{Z}^{0}$ decay at $91.2 \mathrm{GeV}$ with the normal primary flavour mixture.

\section{Gluon splitting to heavy quarks}

Data at LEP1 show a larger rate of secondary charm and bottom production than predicted in most shower descriptions [5, 40], or in analytical studies 41]. We therefore comment on a few of the issues in the Monte Carlo simulation and how a relaxation of some demands would affect rates.

\subsection{Strong coupling argument and kinematics}

The default behaviour in PYTHIA is to let $\alpha_{\mathrm{s}}$ have $p_{\perp}^{2}$ as argument. Actually, the exact kinematics has not yet been reconstructed when $\alpha_{\mathrm{s}}$ is invoked, so the approximate expression $p_{\perp}^{2} \approx z(1-z) m_{a}^{2}$ is used, see discussion at eq. (7). Since $\alpha_{\mathrm{s}}$ blows up when its argument approaches $\Lambda_{\mathrm{QCD}}$, this translates into a requirement on $p_{\perp}^{2}$ or on $z$ and $m_{a}$, restricting allowed emissions to $p_{\perp}>Q_{0} / 2$, where $Q_{0}$ is the shower cut-off scale. Also when full kinematics is reconstructed, this is reflected in a suppression of branchings with small $p_{\perp}$. Therefore, in $g \rightarrow \mathrm{q} \overline{\mathrm{q}}$ branchings analyzed in the g rest frame, the quarks do not come out with the $1+\cos ^{2} \theta$ angular distribution (with respect to the direction of motion of the gluon) one might expect away from threshold, or a somewhat more isotropic one closer to threshold, but are rather peaked at $90^{\circ}$ and dying out at $0^{\circ}$ and $180^{\circ}$.

For $\mathrm{g} \rightarrow \mathrm{q} \overline{\mathrm{q}}$ branchings, the soft-gluon results that lead to the choice of $p_{\perp}^{2}$ as scale 42] are no longer compelling, however. One could instead use some other scale that does not depend on $z$ but only on $m_{a}=m_{\mathrm{g}}$, i.e the off-shellness of the branching gluon, and remove the $p_{\perp}$ cut. A reasonable choice, even if not unique, is to use $m_{\mathrm{g}}^{2} / 4$, where the factor $1 / 4$ ensures continuity with $p_{\perp}^{2}$ for $z=1 / 2$. This possibility has been added as a new option.

Actually, the change of $\alpha_{\mathrm{s}}$ argument in itself leads to a reduced $\mathrm{g} \rightarrow \mathrm{q} \overline{\mathrm{q}}$ splitting rate, while the removal of the $p_{\perp}>Q_{0} / 2$ requirement increases it. The net result is a decrease by about $10 \%$, Table 4 . The topologies of the events are changed somewhat, so rates within experimental cuts could be more affected. However, the changes are not as big as might have been expected - see the following. 


\subsection{Coherence}

In the above subsection, it appears as if the $1+\cos ^{2} \theta$ distribution would be recovered in the new $\alpha_{\mathrm{s}}\left(m_{\mathrm{g}}^{2} / 4\right)$ option. However, this neglects the coherence condition, which is imposed as a requirement in the shower that successive opening angles in branchings become smaller. Such a condition actually disfavours branchings with $z$ close to 0 or 1 , since the opening angle becomes large in this limit, eq. (8). It should be noted that the opening angle discussed here is not the true one, but the one based on approximate kinematics, including neglect of masses. More generally, the coherence formalism is not really developed with this kind of configurations in mind, especially not with a heavy quark pair close to threshold.

As a means to exploring consequences, two new coherence level options have thus been introduced. In the first, the $p_{\perp}^{2}$ of a $\mathrm{g} \rightarrow \mathrm{q} \overline{\mathrm{q}}$ branching is reduced by the correct mass-dependent factor, $1-4 m_{\mathrm{q}}^{2} / m_{\mathrm{g}}^{2}$, while the massless approximation is kept for the longitudinal momentum. This is fully within the uncertainty of the game, and no less reasonable than the default. In the second, no angular ordering at all is imposed on $\mathrm{g} \rightarrow \mathrm{q} \overline{\mathrm{q}}$ branchings. This is certainly an extreme scenario, and should be viewed with caution. However, it is still interesting to see what it leads to.

In Section 3.4 another "intermediate" coherence variation of the default "full" one was introduced, affecting the rate of gluon emission off the primary quarks but not the subsequent gluon cascades. This variation has negligible consequences for the secondary quark rate at LEP energies and is not considered further.

It turns out that the decay angle distribution of the gluon is much more distorted by the coherence than by the $\alpha_{\mathrm{s}}$ and kinematics considerations described earlier. Both modifications are required if one would like to have a $1+\cos ^{2} \theta$ shape, however. Also other distributions, like gluon mass and energy, are affected by the choice of options.

The most dramatic effect appears in the total gluon branching rate, however, Table 4 . Already the reduced angular ordering requirement can boost the $g \rightarrow b \bar{b}$ rate by almost a factor of two. The effects are even bigger without any angular ordering constraints at all. It is difficult to know what to make of these big effects. Experimental information on the angular distribution of secondary $\mathrm{c} \overline{\mathrm{c}} / \mathrm{b} \overline{\mathrm{b}}$ pairs might help understand what is going on, but probably that is not possible experimentally. Anyway, the measured (but still uncertain) values are fully bracketed by the range of the models, indicating that there need not be a conflict between theory and experiment.

\section{Summary and Outlook}

We have in this article studied QCD radiation off heavy particles, both based on the calculation of a wide set of first-order matrix elements and by the usage of these matrix elements as input to a parton-shower description of multiple-gluon emission.

The matrix-element calculations provide at least two important insights. One is the significant spin dependence on the rate of gluon emission, given identical kinematical conditions. Some such effects could be expected simply from the different shape of the splitting kernel of a quark and a squark, say, but equally important is the spin of the decaying particle, and the parity of the process (combining that of mother and daughters). The best illustration is the difference between a vector and a scalar colour singlet source each decaying to a pair of squarks, where the gluon emission rate differs by up to a factor of 1.8 in our limited study, and is likely to reach even higher levels in other observables. 
The $\gamma_{5}$ dependence disappears in the massless limit, but the bulk of other effects remain also there, so this is not only an issue for the production of heavy flavours. This process dependence would not be caught by the traditional parton-shower philosophy, where the universal aspects of gluon emission are emphasized.

The other insight is that the 'dead cone' concept is one that only applies universally for soft gluon emission. Only one process provides an exact zero in the collinear emission rate while, for reasonably hard gluons, some processes show no dip at all at small angles. And, while soft gluons may be ideologically interesting, the experimentally observable event shapes are often more crucially dependent on some intermediate range of gluon energies. Again, therefore, the message is that a universal shower description may be misleading.

Fortunately, it is not impossible to combine the shower picture with a process dependence of the kind noted above. We have in this article developed one approach to the problem, wherein matrix element information is provided for all shower branchings of the primary particles. This combines a process dependent resummation of gluon emission off these particles with the traditional strengths of the shower formalism, such as providing exclusive final states with exact energy-momentum conservation.

To illustrate this formalism, a few physics examples have been studied. For bottom production at the $\mathrm{Z}^{0}$ pole, detailed comparisons with data are possible. We find the new shower routine to be in good agreement with current data if the uncertainties in both model and data are taken into account. A significant dependence on coherence options were found both for jet rates and gluon splitting rates in the shower. It has further been possible to rule out an older version of the shower routine where mass effects were not correctly accounted for.

Differences in jet rates were found for different coupling structures in Higgs decay to bottom, whereas the influence on the fragmentation function is minor. Both the production and decay of the top quark is dependent on the matrix element correction, influencing e.g. the mass reconstruction of the top quark. Again the jet rate was found to be most sensitive.

Gluon radiation off supersymmetric particles, squarks and gluinos, has been introduced, but the effect of this additional radiation is small in high energy processes, especially at hadron colliders, because of the background from initial state radiation and showers off the standard model decay products. Differences between top and stop events are small if equal masses are assumed, but many more comparisons between standard model and supersymmetric processes could be envisaged.

From this limited study we conclude that gluon emission off $\mathrm{b}$ and lighter quarks dominate the picture. If this component is modelled well, the rest is less important, but still of ideological interest. Since the scaled bottom mass $r=m_{\mathrm{b}} / m_{X}$, in the decay of a resonance $X$, is small in most cases, we see little if any parity dependence. In $\mathrm{Z}^{0}$ decays this effect is less than $1 \%$, but it could be visible in a high-precision study. Also for the spin dependence, effects are small in most cases, although the $\mathrm{H}^{0} \rightarrow \mathrm{b} \overline{\mathrm{b}}$ example shows they need not be quite as small. It thus turns out that the new process dependence will mainly be important when high precision is strived at.

We note that the current study is not the end of the story. While we have sampled a fair selection of colour and spin structures, more are likely to turn up even in the simple context of two-body decays considered here. The MSSM also allows a significant rate for three-body decays, normally as a sequence of two consecutive two-body ones with an intermediate off-shell propagator, and with the possibility of interference between several 
such intermediate states. The real challenge, however, may well be provided by the more complex production processes at hadron colliders, where the concept of a sequence of $s$-channel processes need no longer be valid, e.g. squark production gg $\rightarrow \tilde{\mathrm{q}} \overline{\tilde{q}}$ with a $t$ channel squark propagator. The large rate of initial-state radiation also implies that the initial-final state interference terms may be more important than the final-state radiation off the heavy particles themselves. Much work therefore lies ahead, if one desires a good description of QCD effects in many processes of interest at the LHC.

\section{References}

[1] B. Andersson, G. Gustafson, G. Ingelman and T. Sjöstrand, Phys. Rep. 97 (1983) 31 ;

T. Sjöstrand, Nucl. Phys. B248 (1984) 469.

[2] Yu.L. Dokshitzer, V.A. Khoze, L.H. Orr and W.J. Stirling, Nucl. Phys. B403 (1993) 65.

[3] M. Bengtsson and T. Sjöstrand, Phys. Lett. B185 (1987) 435, Nucl. Phys. B289 (1987) 810.

[4] Yu.L. Dokshitzer, V.A. Khoze and S.I. Troyan, J. Phys. G17 (1991) 1602.

[5] A Ballestrero et al., hep-ph/0006259, to appear in the Proceedings of the LEP2 Monte Carlo Workshop.

[6] T. Sjöstrand, Comput. Phys. Commun. 82 (1994) 74, LU TP 95-20 (hep-ph/9508391).

[7] G. Marchesini and B.R. Webber, Nucl. Phys. B238 (1984) 1;

G. Marchesini, B.R. Webber, G. Abbiendi, I.G. Knowles, M.H. Seymour and L. Stanco, Comput. Phys. Commun. 67 (1992) 465.

[8] G. Marchesini and B.R. Webber, Nucl. Phys. B330 (1990) 261.

[9] G. Gustafson and U. Pettersson, Nucl. Phys. B306 (1988) 746;

L. Lönnblad, Comput. Phys. Commun. 71 (1992) 15.

[10] V.V. Sudakov, Zh. E.T.F. 30 (1956) 87.

[11] Workshop on Photon Radiation from Quarks, ed. S. Cartwright, CERN 92-04.

[12] M.H. Seymour, Z. Phys. C56 (1992) 161, Comput. Phys. Commun. 90 (1995) 95.

[13] G. Corcella and M.H. Seymour, Phys. Lett. B442 (1998) 417.

[14] J. André and T. Sjöstrand, Phys. Rev. D57 (1998) 5767.

[15] J. Ellis, M.K. Gaillard and G.G Ross, Nucl. Phys. B111 (1976) 253.

[16] B.L. Ioffe, Phys. Lett. 78B (1978) 277.

[17] A.H. Mueller, Phys. Lett. 104B (1981) 161;

B.I. Ermolaev and V.S. Fadin, JETP Lett. 33 (1981) 269. 
[18] W. Beenakker, R. Höpker and P.M. Zerwas, Phys. Lett. B349 (1995) 463.

[19] Ya.I. Azimov, Yu.L. Dokshitzer, V.A. Khoze and S.I. Troyan, Phys. Lett. 165B (1985) 147.

[20] B. Andersson, G. Gustafson and T. Sjöstrand, Phys. Lett. 94B (1980) 211.

[21] V.A. Khoze and T. Sjöstrand, Phys. Lett. B328 (1994) 466.

[22] G. Gustafson, U. Pettersson and P. Zerwas, Phys. Lett. B209 (1988) 90;

T. Sjöstrand and V.A. Khoze, Z. Phys. C62 (1994) 281, Phys. Rev. Lett. 72 (1994) 28 ;

L. Lönnblad and T. Sjöstrand, Phys. Lett. B351 (1995)293, Eur. Phys. J. C2 (1998) 165.

[23] CDF Collaboration, F. Abe et al., Phys. Rev. D50 (1994) 5562.

[24] E. Norrbin and T. Sjöstrand, Phys. Lett. B442 (1998) 407, LU TP 00-16 hepph/0005110), to appear in Eur. Phys. J. C.

[25] A. Pukhov et al., preprint INP MSU 98-41/542 (hep-ph/9908288).

[26] A. Arhib, M.C. Peyranere and G. Moultaka, Phys. Lett. B341 (1995) 313.

[27] Wolfram Research, Inc., MathematicA, Version 2.2, Champaign, IL (1993).

[28] P. Janot, Phys. Lett. B223 (1989) 110.

[29] J. Jersak, E. Laermann and P.M. Zerwas, Phys. Lett. 98B (1981) 363;

M. Jeżabek and J.H. Kühn, Nucl. Phys. B314 (1989) 1.

[30] R.K. Ellis, D.A. Ross and A.E. Terrano, Nucl. Phys. B178 (1981) 421.

[31] Z. Kunszt, Phys. Lett. 99B (1981) 429.

[32] S. Catani, Yu.L. Dokshitzer, M. Olsson, G. Turnock and B.R. Webber, Phys. Lett. B269 (1991) 432.

[33] T.R. Grose and K.O. Mikaelian, Phys. Rev. D23 (1981) 123.

[34] Yu.L. Dokshitzer, V.A. Khoze, A.H. Mueller and S.I. Troyan, Rev. Mod. Phys. 60 (1988) 373.

[35] JADE Collaboration, W. Bartel et al., Z. Phys. C33 (1986) 23;

JADE Collaboration, S. Bethke et al., Phys. Lett. B213 (1988) 235;

N. Brown and W.J. Stirling, Z. Phys. C53 (1992) 629;

Y.L. Dokshitzer, G.D. Leder, S. Moretti and B.R. Webber, JHEP 9708 (1997) 001;

L. Lönnblad, S. Moretti and T. Sjöstrand, JHEP 9808 (1998) 001.

[36] Delphi Collaboration, P. Abreu et al., Phys. Lett. B418 (1998) 430;

P. Bambade, private communication.

[37] Aleph Collaboration, R. Barate et al., CERN-EP/2000-093 (hep-ex/0008013). 
[38] A.H. Hoang et al., Eur. Phys. J. direct C3 (2000) 1 (hep-ph/0001286), to appear in the proceedings of the workshop "Physics Studies for a Future Linear Collider", Top Quark WG.

[39] S. Mrenna, Comput. Phys. Commun. 101 (1997) 232.

[40] M.L. Mangano, in International Europhysics Conference on High Energy Physics, eds. K. Huitu et al. (IOP Publishing, Bristol, 2000), p. 33.

[41] M.H. Seymour, Nucl. Phys. B436 (1995) 163;

D.J. Miller and M.H. Seymour, Phys. Lett. B435 (1998) 213.

[42] D. Amati, A. Bassetto, M. Ciafaloni, G. Marchesini and G. Veneziano, Nucl. Phys. B173 (1980) 429. 